\title{
Article \\ Central Attention and a Dual Path Convolutional Neural Network in Real-World Tree Species Recognition
}

\author{
Yi Chung ${ }^{1, * \mathbb{D}}$, Chih-Ang Chou ${ }^{2}$ and Chih-Yang $\mathrm{Li}^{3}$ \\ 1 College of Human Development and Health, National Taipei University of Nursing and Health Sciences, \\ Taipei 11219, Taiwan \\ 2 Xin Ji International Company, New Taipei 234014, Taiwan; catchsob@gmail.com \\ 3 Department of Computer Science and Information Engineering, National Taiwan University, \\ Taipei 10617, Taiwan; taipingeric@gmail.com \\ * Correspondence: m9306009@gmail.com
}

check for

updates

Citation: Chung, Y.; Chou, C.-A.; Li, C.-Y. Central Attention and a Dual Path Convolutional Neural Network in Real-World Tree Species Recognition. Int. J. Environ. Res. Public Health 2021, 18, 961. https:// doi.org/10.3390/ijerph18030961

Received: 2 December 2020

Accepted: 14 January 2021

Published: 22 January 2021

Publisher's Note: MDPI stays neutral with regard to jurisdictional claims in published maps and institutional affiliations.

Copyright: (c) 2021 by the authors. Licensee MDPI, Basel, Switzerland. This article is an open access article distributed under the terms and conditions of the Creative Commons Attribution (CC BY) license (https:// creativecommons.org/licenses/by/ $4.0 /)$.

\begin{abstract}
Identifying plants is not only the job of professionals, but also useful or essential for the plant lover and the general public. Although deep learning approaches for plant recognition are promising, driven by the success of convolutional neural networks $(\mathrm{CNN})$, their performances are still far from the requirements of an in-field scenario. First, we propose a central attention concept that helps focus on the target instead of backgrounds in the image for tree species recognition. It could prevent model training from confused vision by establishing a dual path CNN deep learning framework, in which the central attention model combined with the CNN model based on InceptionV3 were employed to automatically extract the features. These two models were then learned together with a shared classification layer. Experimental results assessed the effectiveness of our proposed approach which outperformed each uni-path alone, and existing methods in the whole plant recognition system. Additionally, we created our own tree image database where each photo contained a wealth of information on the entire tree instead of an individual plant organ. Lastly, we developed a prototype system of an online/offline available tree species identification working on a consumer mobile platform that can identify the tree species not only by image recognition, but also detection and classification in real-time remotely.
\end{abstract}

Keywords: plant recognition; deep learning; dual path convolutional neural network; visual attention; mobile application

\section{Introduction}

\subsection{Motivation}

Plants are important resources on Earth to maintain ecosystems, promote the medical sector, and increase agricultural productivity and sustainability [1]. Moreover, regional biodiversity studies, population size estimates of endangered species, and species distribution due to climate change depend on the accuracy of plant identification [2].

One of the most obvious features of organic life is its remarkable diversity [3]. Despite the variation of organisms, a more experienced eye soon distinguishes that organisms can be grouped into taxa [4]. These days, with the continuous loss of biodiversity [5], the demand for routine species identification has risen; meanwhile, the number of experienced experts is limited and reduced [6].

Nowadays, the growth of technology and urbanization has decreased the knowledge and uses of plants by humans. However, the environment and the natural resources raise a growing concern. An accurate automated identification system enables non-experts with only limited botanical training and expertise to contribute to the survey of the world's biodiversity [4], thus providing inexperienced people, who had no knowledge in botany, with a tool to recognize the plants that surround them would be a great advance [7]. 
Plant identification is not only the work of botanists and ecologists, but also useful or essential for large segments of society, from professionals to the general public. However, the identification of plants by conventional ways is difficult, time consuming, and frustrating for novices due to the use of specific botanical terms [4].

Recently, computer science research, especially image processing and pattern recognition techniques, have been proposed to plant taxonomy in order to eventually overcome the deficiency in human identification abilities [4]. Field researchers, land managers, civil servants, educators, and the interested public would greatly benefit from up-to-date and accessible tools automating the process of species identification. Notably, relevant technologies, such as mobile devices, digital cameras, and remote access to databases, are ubiquitously available, accompanied by significant advances in computer vision [4]. With the popularity of smartphone devices, web-based and stand-alone applications have been developed to help users identify plant species [8].

\subsection{Deep Learning}

Deep learning approaches have shown promising results in various computer vision problems, including the plant identification task driven by the success of convolutional neural networks (CNN) [4]. The essence of deep learning is its capacity to create and extrapolate new features from raw representations of input data without having to be told explicitly which features to use and how to extract them [9].

The latest studies on plant identification utilize these techniques and achieve significant improvements over methods developed in the decade before. However, a few of them were conducted in controlled environments, with lighting and background being constants that limited the flexibility of user experiences [4].

\subsection{Challenges in Real-World Scenarios}

The training dataset is the cornerstone, upon which deep learning algorithms depend [10]. There were some plant species benchmark datasets, most of which were leaf images scanned or photographed with plain background such as Swedish leaf [11], Flavia [12], Leafsnap [13], and Intelligent Computing Laboratory (ICL) [14]. The Plant Cross Language Evaluation Forum (PlantCLEF) [15] dataset contained more realistic images, such as multiple organs like fruits, flowers, leaves, and stems in the natural environment. However, the collected species were mainly focused on the Guiana shield and the Northern Amazon rainforest, and the average number of image per species was three times less than the previous edition of PlantCLEF; some species may even contain only one image.

In fact, most botanists sometimes simultaneously observe several organs of the same plant in order to disambiguate species easily confused when observing only a single organ [16]. A plant classification method is presented using a combination of leaf, flower, and bark photos of the same tree species, and achieves recognition accuracy on multi-organ better than the accuracy on a single organ [17]. Rather than a full view of the entire plant, a single organ photograph could lead to the high risk of recognition errors because of disproportion to the real world. Additionally, these previous bench datasets were limited in the number of species due to the tremendous effort for collecting them, especially for biodiversity in regional differences. Plant recognition is still challenging due to the great variability of these characteristics within images from the same species and also the similarity of some characteristics between different species [18].

Here, we proposed a comprehensive study of deep CNNs to extract the feature vectors from the images of entire plant observations.

\subsection{Central Attention}

The background of photos taken in real-world ways is usually complicated. A realworld plant image contains more than one object, i.e., target plants and other interferents in the background. Furthermore, target plants are possibly touching or overlapped with the background objects. However, the salient objects what we pay attention to are to be 
recognized in an image. For an image, human visual attention allows us to rapidly locate the most important information in a scene [9], and the most useful point is focused on with our attention at first sight for a given object [10].

In addition to the interference of the non-target, there are also non-valuable redundancy. The object is recognized only with the sketchy and concentrated screenage or information born in our mind although there is a large amount of content. Other nonsalient parts are ignored or neglected. Therefore, we are not even aware of the redundancy during the first judgement [19].

Xiao et al. proposed a deep learning framework with attention cropping [19]. The input images were cropped in terms of visual attention before recognized. Attention cropping can focus on the real interesting target and remove the interferences, and was accomplished with the generated saliency map using the saliency detection approach [20]. Results show that the targets and foregrounds were reserved and the distant surroundings and redundancies were tailored out after attention cropping. The final identification was made using pre-trained deep CNNs. The results of CNNs using attention cropping augmentation were superior to those of $\mathrm{CNNs}$ without attention cropping. InceptionV3 with attention cropping outperformed classic InceptionV3 by $4.2 \%$ accuracy.

Visual attention prediction aims to predict scene locations where a human observer may fixate [21]. Recently, driven by the success of deep learning in object recognition, many deep learning based attention models [22-25] were proposed, and generally gave impressive results. The attention-based approaches [26-28] focus on preserving the main subject or visually important area in the scene after cropping [21]. These methods usually place the crop window over the most visually significant regions according to certain attention scores [29-32].

The most important information and what we are most interested are facilitated to be located using attention cropping to fulfill real-world identification although there are different scenarios and the background is complex in realistic images. Notably, attention cropping possesses greater advantage and better performance for real-world recognition compared with the conventional recognition where the background is simple [19]. Furthermore, when taking pictures, users tend to place the subject in the middle according to our observation and survey.

Based on this attention concept and the user experience observation, we defined the salient objects which are to be recognized in an image are focused in the center. We cropped the image from the center, acquired it as the input of one CNN path, and named the operation central attention.

\subsection{Dual-Path CNN}

Several dual path CNN models were developed in multiple domains, and generally showed remarkable results [33-37]. In the present study, we constructed a dual path CNN, one of which is the central attention path while the other is a classic CNN path. The backbone architecture of the former path is a classic CNN path with inputs from the central attention cropping images while the later one is a classic $\mathrm{CNN}$ path with inputs from the original images.

\subsection{Mobile Application}

With the popularization of intelligent mobile devices, the automated plant identification technology on the edge side plays a crucial role in the fields of ecological environment supervision, ecological science popularization, and foreign plant invasion monitoring [38].

A smartphone consists of everything required for the implementation of a mobile plant identification system, including a camera, a processor, a user interface, and an Internet connection. These requirements make smartphones particularly suitable for field use by professionals and the general public. However, these devices still have less storage capacity, available memory, network bandwidth, and computational power than desktop computers or servers, limiting algorithmic choices. Due to these limitations, it can be 
tempting to offload some of the processing to a high-performance server that requires a reliable Internet connection. Using an online service can be attractive when dataset or algorithm is likely to be updated regularly or when they have large computational and memory requirements. However, in remote areas where plant identification applications are likely to be most useful, an Internet connection may be unavailable or unreliable. The alternative approach is to use efficient algorithms that run directly on the device without the need for a network connection or a support server but with potential limitations in the classification performance $[39,40]$.

Xingse [41], a mobile application for plant recognition developed in China, has not documented several endemic plant species in Taiwan even with similar environmental conditions, such as Millettia pinnata, not to mention some of the well-known mobile apps [42-44] that were sampled outside of Asia may result in insufficient plant species in their database due to regional differences.

Numerus automatic systems were designed for plant identification, but only a few mobile applications can be used simply to identify tree species just by clicking on a button slightly or moving around the mobile camera with real-time object detection and classification. Up to now, the latest developments about the automatic identification system was presented. However, these studies were mostly limited to leaf images [45]. To solve these problems, we aim to develop a tree recognition mobile application with a superior user experience which is simpler, intuitive, and much more accurate.

\subsection{The Aim of the Study}

We aim to introduce the central attention concept implemented with a dual path CNN for real-world tree species recognition. For application, the purpose of this study is to provide the general public with better user experiences in recognizing tree species, so as to reduce barriers to entry for the ecological filed, while offering the plant lover a seamless user experience to assist one in identifying confusing tree species and improving the recognition efficiency.

To begin with, we collect our own dataset containing 14 common tree species in Taiwan with the images of entire plant observations by taking pictures in the field. Then, we introduce a central attention concept which is constructed in a dual path deep learning framework based on the CNN, trained the model by using the self-collected dataset, and underwent the test of identifying the trees based on their morphological features. Furthermore, in order to verify the validity of the proposed framework, we used a uni-path traditional CNN and a uni-path central attention method to perform the same experiment for comparative analysis. Experiments showed that our proposed dual path deep learning framework had achieved good effect of identification. Finally, we implemented the tree species recognition into an Android mobile application, which can detect object with mobile cameras in real-time, display the label, and overlay on the camera image. No Internet connection is required for using this app.

\subsection{Related Works}

\subsubsection{Deep learning}

Deep learning related works were summarized in Table 1. Different modern and advanced models have been proposed for automated plant recognition as the deep learning technology advances [1].

One of the first studies on plant identification using a CNN is Lee et al's leaf classifier which utilized the AlexNet model pre-learned on the ImageNet Large Scale Visual Recognition Challenge 2012 (ILSVRC2012) dataset and reported an average accuracy of 99.7\% on the MalayaKew (MK) leaf dataset consisting of 44 species [9,46]. TreeID, a plant species identification system, was developed with a simple three-layer CNN [47]. Wu et al. used a four-layer CNN for classification of leaves accompanied by the Parametric Rectified Linear Unit (PReLU) activation function instead of traditional Rectified Linear Unit (ReLU), and were able to obtain an accuracy of $94.8 \%$ on the ICL dataset with 50 different species of leaves [48]. 
Table 1. Summary of related works in deep leaning.

\begin{tabular}{|c|c|c|c|c|}
\hline Authors, Year & Image Type & Dataset & Method & Accuracy \\
\hline $\begin{array}{l}\text { Lee et al., 2015, } \\
2017[9,46]\end{array}$ & Leaf & $\begin{array}{l}\text { MalayaKew Leaf } \\
\text { dataset }\end{array}$ & $\begin{array}{l}\text { Convolutional Neural Network } \\
(\mathrm{CNN})+\text { AlexNet }\end{array}$ & $99.7 \%$ \\
\hline Chopra, 2015 [47] & Leaf & TreeID & Three-layer CNN & $75 \%$ \\
\hline Wu et al., 2016 [48] & Leaf & ICL & $\begin{array}{l}\text { Four-layer CNN + PReLU } \\
\text { activation function }\end{array}$ & $94.8 \%$ \\
\hline Bao et al., 2019 [49] & Leaf & $\begin{array}{l}\text { Flavia and Swedish } \\
\text { leaf dataset }\end{array}$ & $\begin{array}{l}\text { Histogram of oriented gradients } \\
(\mathrm{HOG})+\text { support vector machine } \\
(\mathrm{SVM}) \text { classifier + five-layer CNN }\end{array}$ & $95.6 \%$ \\
\hline Zhang et al., 2015 [50] & Leaf & Flavia & Seven-layer CNN & $94.69 \%$ \\
\hline Barre et al., 2017 [51] & Leaf & $\begin{array}{l}\text { LeafSnap, Flavia, and } \\
\text { Foliage datasets }\end{array}$ & 17-layer CNN & $97.9 \%$ \\
\hline Zhu et al., 2018 [45] & $\begin{array}{l}\text { Leaf, flower, } \\
\text { fruit, branch, } \\
\text { stem }\end{array}$ & LifeCLEF2015 dataset & 19-layers CNN + SVM & $\begin{array}{l}\text { Leaf }(67.10 \%) \text {, flower } \\
(88.80 \%), \text { fruit }(90.20 \%), \\
\text { branch }(71.20 \%), \\
\text { stem }(65.20 \%)\end{array}$ \\
\hline \multirow[t]{2}{*}{$\begin{array}{l}\text { Pearline et al., } \\
2019 \text { [52] }\end{array}$} & \multirow[t]{2}{*}{ Leaf } & Leaf12 dataset & $\begin{array}{l}\text { VGG } 16 \text { CNN architecture with } \\
\text { logistic regression (LR) }\end{array}$ & $97.14 \%$ \\
\hline & & $\begin{array}{l}\text { Folio, Flavia, and } \\
\text { Swedish leaf datasets }\end{array}$ & $\begin{array}{l}\text { VGG } 19 \text { CNN architecture with } \\
\text { LR classifier }\end{array}$ & $\begin{array}{l}\text { Folio }(96.53 \%) \text {, Flavia } \\
(96.25 \%) \text { Swedish } \\
(99.41 \%)\end{array}$ \\
\hline Goëau et al., 2019 [15] & Plant & $\begin{array}{l}\text { LifeCLEF2019 plant } \\
\text { dataset }\end{array}$ & $\begin{array}{l}\text { InceptionV4 and Inception-ResNet-v2 } \\
\text { CNN }\end{array}$ & $31.6 \%$ \\
\hline Sun et al., 2017 [53] & Leaf & $\begin{array}{l}\text { Flavia, BJFU100 } \\
\text { datasets }\end{array}$ & 26-layer ResNet & $\begin{array}{l}\text { Flavia }(99.65 \%) \\
\text { BJFU100 }(91.78 \%)\end{array}$ \\
\hline $\begin{array}{l}\text { Bodhwani et al., } \\
2019 \text { [1] }\end{array}$ & Leaf & LeafSnap & $\begin{array}{l}\text { 50-layer deep residual } \\
\text { learning framework }\end{array}$ & $93.09 \%$ \\
\hline
\end{tabular}

Bao et al. compared a traditional shallow architecture, which extracted a feature histogram of an oriented gradients (HOG) vector along with an Support Vector Machine (SVM) classifier, to a deep five-layer CNN on the Flavia and Swedish leaf datasets. The deep architecture presented greater accuracy, and worked well on classification problem of leaves based on the shape of veins [49]. Zhang et al. utilized a seven-layer CNN to classify the Flavia dataset, and reached $94.69 \%$ accuracy [50]. Barre et al. further improved this result by using a 17-layer CNN and obtained an accuracy of $97.9 \%$ validated on the LeafSnap, Flavia, and Foliage datasets [51].

Zhu et al. employed a 19-layer CNN in combination with a linear classifier SVM, and outperformed conventional methods based on hand-crafted features [45]. Pearline et al. utilized Visual Geometry Group 16 (VGG 16) CNN architecture with logistic regression (LR) that resulted in an accuracy of $97.14 \%$ for Leaf12 dataset, while the VGG 19 CNN architecture with a logistic regression classifier reached an accuracy of $96.53 \%, 96.25 \%$ and 99.41\% on Folio, Flavia, and Swedish leaf datasets, respectively. Moreover, they found that VGG 16 or 19 deep learning architectures with an LR classifier resulted in higher accuracy compared with InceptionV3 and Inception-ResNet-v2. [52]. However, InceptionV4 and Inception-ResNet-v2 CNN models won the ExpertLifeCLEF Plant Identification Task 2018 and LifeCLEF Plant Identification Task 2019 [15,54].

Sun et al. studied the ResNet architecture, which solves the vanishing gradient and degradation problems [55]. They found a 26-layer network to achieve best performance with $99.65 \%$ on the Flavia dataset with a simple background, and $91.78 \%$ on Beijing Forestry University 100 (BJFU100) dataset, which consists of 100 species of ornamental plants multiplied by 100 photos in natural scenes [53]. Eventually, Bodhwani et al. designed a 50-layer deep residual learning framework, and achieved a recognition rate of $93.09 \%$ 
with $0.24 \%$ error on the LeafSnap dataset [1]. In spite of intensive and elaborate studies on automated plant species identification, only a little research resulted in approaches which can be used by the general public [4].

\subsubsection{Mobile Applications}

Mobile Applications related works were summarized in Table 2. LeafView [56], a tablet PC-based application, can be used offline for the automated identification of species directly in the field. A user took a picture of a single leaf on a blank background, and the app extracted the shape feature and matched it with the existing species. The system then showed the top matches in a few seconds with text descriptions and additional photographs, or marked unknown for further study. The system returned the correct species in the top ten matches $90-97 \%$ of the time.

Table 2. Summary of related works in mobile applications.

\begin{tabular}{|c|c|c|c|c|}
\hline Authors, Year & Image Type & Mobile Applications & Method & Accuracy \\
\hline $\begin{array}{l}\text { Belhumeur et al., } \\
2008 \text { [56] }\end{array}$ & Leaf & LeafView & Nearest neighbor $(\mathrm{NN})$ classifier & $\begin{array}{l}\text { Top ten matches } \\
90-97 \%\end{array}$ \\
\hline Kumar et al., [42] & Leaf & Leafsnap & $\mathrm{NN}$ & $73 \%$ \\
\hline Goëau et al., 2013 [43] & $\begin{array}{l}\text { Leaf, flower, } \\
\text { fruit and bark }\end{array}$ & Pl@ntNet & $\begin{array}{l}\text { Approximate k-NN search }+ \\
\text { administration of material purchases } \\
\text {-local sensitive hashing (AMP-LSH) }\end{array}$ & $\begin{array}{l}69 \% \text { top- } 5 \\
\text { identification } \\
\text { rate }\end{array}$ \\
\hline Cerutti et al., 2013 [57] & Leaf & Not available (N/A) & $\begin{array}{l}\text { Linear regression }(\mathrm{LR})+\text { adaptive local } \\
\text { mean }(\mathrm{AM})\end{array}$ & $\mathrm{N} / \mathrm{A}$ \\
\hline Ma et al., 2013 [58] & Leaf & ApLeafis & $\begin{array}{l}\text { Content-based image retrieval (CBIR) } \\
\text { HSV (Hue, Saturation, Value), Wavelet, } \\
\text { pyramid histogram of orientated } \\
\text { gradients (Phog) + tophat + cropped }\end{array}$ & $90 \%$ \\
\hline $\begin{array}{l}\text { Nguyen et al., } \\
2013 \text { [59] }\end{array}$ & Leaf & $\mathrm{N} / \mathrm{A}$ & $\begin{array}{l}\text { Speeded Up Robust Features (SURF) + } \\
\text { Bag of Words (BOW) + support vector } \\
\text { machine (SVM) }\end{array}$ & $95.94 \%[60]$ \\
\hline Prasad et al., 2013 [61]. & Leaf & $\mathrm{N} / \mathrm{A}$ & k-NN classifier & $70.09-91.34 \%$ \\
\hline Wang et al., 2013 [40]. & Leaf & $\mathrm{N} / \mathrm{A}$ & $\begin{array}{l}\text { Multiscale shape descriptor based on the } \\
\text { concave and convex measures }+\mathrm{k}-\mathrm{NN}\end{array}$ & $86.86-96.05 \%$ \\
\hline $\begin{array}{l}\text { Priyankara et al., } \\
2015 \text { [62] }\end{array}$ & Leaf & $\mathrm{N} / \mathrm{A}$ & $\begin{array}{l}\text { Scale Invariant Feature Transform (SIFT) } \\
+ \text { BOW feature vector + SVM classifier. }\end{array}$ & $96.48 \%$ \\
\hline Zhao et al., 2015 [63] & Leaf & ApLeaf & HSV, Wavelet, Phog + tophat + cropped & $90 \%$ \\
\hline $\begin{array}{l}\text { Van Horn et al., } \\
2017[44]\end{array}$ & $\begin{array}{l}\text { Plant and } \\
\text { animal }\end{array}$ & iNaturalist & $\begin{array}{l}\text { Inception ResNetV2 + } \\
\text { Squeeze-and-Excitation (SE) blocks }\end{array}$ & $67.3 \%$ \\
\hline
\end{tabular}

Leafsnap [42] is the first widely distributed Android classification application. Implemented as a mobile app, it used computer vision techniques for identifying tree species from photographs of their leaves on a simple background. Based on leaf contour features, the app utilized integral measure to compute functions of the curvature at the boundary over multiple scales for classification [64], and retrieved leaf images similar to the query by nearest neighbors $(\mathrm{NN})$ for identification [46]. Then, it was up to the user to make the final decision on what species matched the unknown one. LeafSnap achieved a top-1 recognition rate of $73 \%$ and a top-5 recognition rate of $96.8 \%$ for 184 tree species [65]. The app had attracted a considerable number of downloads, but also received many critical reviews [66] because it fails to handle cluttered backgrounds and does not pay enough attention to both large intra-species visual variations and strong inter-species visual similarities which are typical in the botanical domain [4]. Although efficient for a single input, its processing time hindered the classification of larger sets with a rate of $5.4 \mathrm{~s}$ per sample [33]. Moreover, it 
can make the identification only with access to the Internet, the query image must be taken by the local camera, and is restricted to tree species of the Northeastern United States [39].

Pl@ntNet [43] is an image sharing and retrieval application for botanical identification, available on three front-ends, an iOS app, an Android app, and a web interface. Each allowed users to submit one or several images of a plant with the intention of obtaining a list of the most likely species. Pl@ntNet adopted deep learning techniques for image classification pre-trained on the ImageNet dataset and periodically fine-tuned on steadily growing Pl@ntNet data since 2015 [67]. The application has been downloaded by more than 5 million users worldwide. Joly et al. [68] evaluated the application which supported the identification of 2200 species at that time, and reported a $69 \%$ top-5 identification rate for single images [4].

FOLIA [57], an interactive iOS application, helps to identify a plant species in the natural environment. In order to perform this function, the software first lets the user photograph an unrecognized plant with a smartphone camera. It then extracts high-level morphological characteristics to determine the list of the best matches.

ApLeafis [58] is an Android-based plant leaf identification system in JAVA based on content-based image retrieval technique. An isolated leaf needs to be properly shot on a light, plain, and uniform background. In contrast to Leafsnap, ApLeafis can function without an Internet connection, and select digital images as query images.

Similar to Leafsnap, a leaf based plant identification system for Android used a client-server implementation was developed. To begin with, a leaf photo was taken with the phone, and then being sent to the server for analysis in order to identify the species. Furthermore, the server processed two steps. First was to check if the uploaded photo was a leaf. Then, if the image was validated containing a leaf, the species identification would be activated; otherwise the system would ask for another photo. The identification method was based on Speeded Up Robust Features combined with Bag of Words (BOW) and supervised learning. Finally, the species information would be displayed to the user. The results showed that the method obtained the $95.94 \%$ average accuracy [59].

A low computational approach was proposed with an offline mobile application for Android using OpenCV [61]. Leaf images were captured with the device's camera and must have a uniform background in order to simplify the segmentation. The shape and color features were extracted. The algorithm calculated the geometric feature and then polar Fourier transform, and trained using k-nearest neighbor (k-NN) classifier. After that, two nearest classes were selected on the basic of smallest distance which was further rectified by the color features using a decision tree. The classification process was done on the mobile device itself.

In another Android application in Java, classification can either be done on the server for more computationally expensive algorithms or offline on the device [40]. The online mode involved sending only the feature vector to the server rather than the actual image. The feature extraction was done on the phone itself, significantly reducing bandwidth requirements for the server connection that allowed the most consistent and reliable match speed and continued to function without a network connection, for example, in the wild. Finally, the server returned a dynamic webpage in the device's browser with the closest matches to the database. For the offline recognition mode of the algorithm, the feature extraction, database search and extraction of the top 10 results took between 145-171 ms. The database consisted of $300 \mathrm{~KB}$ of features, and $19 \mathrm{MB}$ of images (or $1.5 \mathrm{MB}$ if only a single image per class was used for display).

An Android client application, which interacts with a leaf recognition algorithm running on the server through a Simple Object Access Protocol-based web service, was developed [62]. The developers used the Scale Invariant Feature Transform (SIFT) algorithm combining with BOW model for feature vector dimensionality reduction, and Support Vector Machine (SVM) classifier. SIFT features were used because they are invariant in scale, rotation, camera viewpoint, and illumination. The BOW model could reduce the high dimensionality of the data space. SVM has a simple structure, comparatively fast speed on 
training, and it is easy to implement. OpenCV was utilized for the actual image processing. The system was trained to classify 20 species and obtained $96.48 \%$ accuracy level.

ApLeaf [63], an Android-based plant leaf identification system, was developed to automatically identify plant species by the photographs of tree leaves. To start with, given a query image taken by the camera or already existed in the local database, the authors segmented it into a binary image by threshold segmentation, and moved the stem by the Tophat method at the preprocessing step. The image should be a single leaf placed on an untextured and light background without other clutter. Next, important features, such as pyramid histograms of oriented gradients and color, were extracted and fused to form the final feature space. Finally, they used the histogram intersection to predict the class and returned to the users the top species which match the query image best. The system was trained on the ImageCLEF2012 Plant Identification database which contains 126 tree species from the French Mediterranean area. It depended on several aspects in computer vision, including segmenting leaves from background, extracting various features by distance measure for species identification, showing users the list of matched species. The performance of the app showed up to $90 \%$ accuracy.

Other similar smartphone applications served as identification aides and/or as repositories of educational information were publicly available [69], such as PlantSnap [70], WildSnap [71], Forest Tree Identification [72], PictureThis—Plant Identifier (the English version of Xingse) [73], About My Woods [74], and Southeast Early Detection Network (SEEDN) [75].

Through deep learning, an iOS mobile application for automated recognition of plants and flowers was introduced [10]. Unlike other apps which focus on static pictures for feature classification, they used video data that compensates for the possibly lost information when comparing a static image with many others images of plants and flowers. This application reported the capability to identify $122 / 125$ plants and $47 / 50$ genera selected with degrees of confidence up to $95 \%$. They also describe the performance speed up through the use of Cloud-based resources.

iNaturalist [44], automatically identified animals and plants at the species level, was launched for Android and iOS by iNaturalist.org in 2017. At first, the app only offered crowd-sourced species identification. Users posted a picture of a plant or animal, and a community of scientists and naturalists identified it. A taxon was raised to "research grade" as soon as more than $2 / 3$ of the involved identifiers agreed in their identification of an observation. It used NVIDIA GPUs (NVIDIA Corporation, Santa Clara, CA, USA) and the Compute Unified Device Architecture (CUDA) deep neural network library along with the TensorFlow deep learning framework. That provided training of the neural networks with an image database which have been labeled by the site's community of experts [76]. iNaturalist already identified over 10,000 different species with a new species added to the model every $1.7 \mathrm{~h}$ [77].

Although numerous progresses have been made in plant identification research, several problems still exist. For example, in the process of leaf identification based on image analysis, the feature of extraction was usually determined by manual analysis. The differences among plant species were not solved, and the differences among plant datasets with the same features would be produced. In the case of complicated background shooting, the identification accuracy of conventional approaches reduced significantly. Furthermore, traditional leaf identification also has some weaknesses that the training image dataset contains too little information about the plants and lacks of complex backgrounds [36]. To this end, we have created a dataset of 14 different trees that contain more complex information about the entire plant.

Overall, most of the related works used merely leaf images with simple, light and untextured background for plant species recognition. Therefore, we aim to recognize tree species by the whole plant images, which not only increase the recognition accuracy, but may enable remote recognition in the natural environment. Although these applications are promising, their performances are still far from the requirements of a real-world ecological 
in-field scenario [39]. Making accurate plant observations from the mass of users requires to equip them with much more accurate identification tools [78].

\section{Method}

\subsection{Dataset}

We created an image dataset of 14 tree species of the most common and endemic in Taiwan, including Koelreuteria henryi, Liquidambar formosana, Ficus microcarpa, Terminalia catappa, Cinnamomum camphora, Delonix regia, Alstonia scholaris, Roystonea regia, Cassia fistula, Bischofia javanica, Melia azedarach, Melaleuca leucadendra, Terminalia mantaly, and Millettia pinnata [79-81]. The raw database contained more than 30,000 photos. After deleting unqualified pictures with redundant, interfering backgrounds and non-targets, the dataset consisted of 2332 field images taken by mobile devices with natural backgrounds, different resolutions, and a wealth of information on the entire tree including leaves, branches, stems, and so on as a whole. It featured visually similar species, captured in a wide variety of situations. The training set contained 1843 images, and the test set included 489 images. A view of the image distribution per selected class was shown in Table 3.

Table 3. Image count per class in the 14 tree species dataset.

\begin{tabular}{cc}
\hline Class Label & Image Count \\
\hline Koelreuteria henryi & 203 \\
Liquidambar formosana & 150 \\
Ficus microcarpa & 295 \\
Terminalia catappa & 62 \\
Cinnamomum camphora & 201 \\
Delonix regia & 105 \\
Alstonia scholaris & 96 \\
Roystonea regia & 68 \\
Cassia fistula & 105 \\
Bischofia javanica & 164 \\
Melia azedarach & 56 \\
Melaleuca leucadendra & 100 \\
Terminalia mantaly & 170 \\
Millettia pinnata & 68 \\
Total image count & 1843 \\
\hline
\end{tabular}

\subsection{Implementation and Preprocess}

The model implementation was based on the open source deep learning framework keras [82]. The algorithm was implemented using Python 3.6.8 (Python Software Foundation, Delaware, USA) on Tensorflow 1.13 (Google LLC, Mountain View, CA, USA) [83], the libraries keras 2.3.0. (Google LLC, Mountain View, CA, USA), and run on Windows 10 (Microsoft Corporation, Redmond, WA, USA) with a NVIDIA GeForce 1070 GPU (NVIDIA Corporation, Santa Clara, CA, USA) for deep learning. Image preprocessing included transforming each image to gray-scale, then applying Gaussian filters, and lastly thresholding. All input image size in our CNN backbone model was resized to $448 \times 448$ pixels, and in our central attention model was cropped from the center, resized into $224 \times 224$ pixels. Each image was fed to the model as a full re-sized image and a center-cropped image, for the $\mathrm{CNN}$ backbone and central attention paths accordingly. Then, the per-pixel value was divided by 255 , and all samples were shuffled for training [84].

We augmented the dataset using the following affine transformations to reach a dataset of 4000 samples. These transformations were label-preserving. The used transformations were random rotations of the image by an angle of up to 10 degrees; vertical or horizontal translation by a distance within 0.1 of the patch size randomly; horizontal flip: randomly flipping half of the images horizontally; and zooming inside pictures in the range $0.5-1.5$ randomly.

\subsection{Central Attention}

As shown in Figure 1, interfering backgrounds, such as buildings, cars, street lights, pavement roads, and so on, could risk a mis-trained tree recognition model demonstrated by 
Gradient-weighted Class Activation Mapping (Grad-CAM) [82]. In addition, users tend to take photos with the subject in the center according to the observation and survey on user experiences. Therefore, in order to prevent the interference, we suggest focusing on the middle part of the full picture by cropping the center half of the image as illustrated in Figure 2.
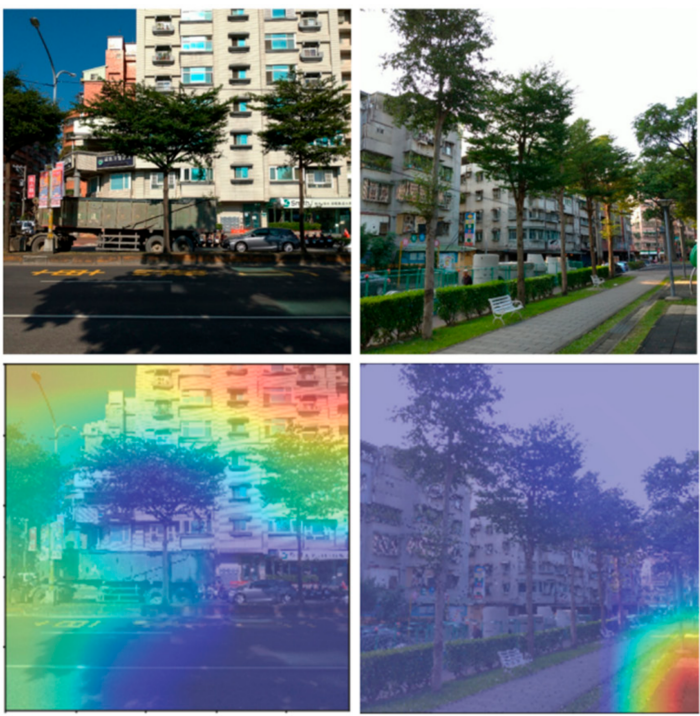
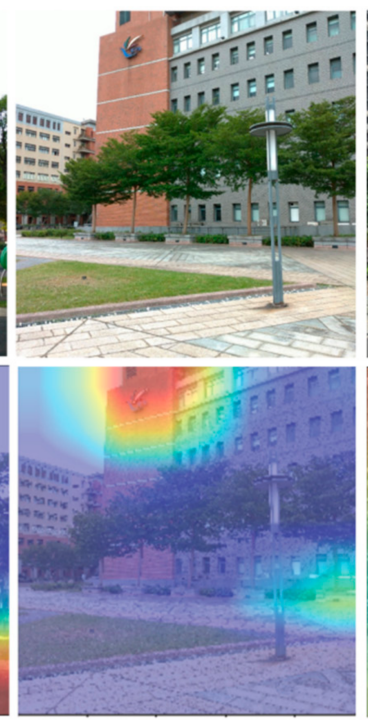
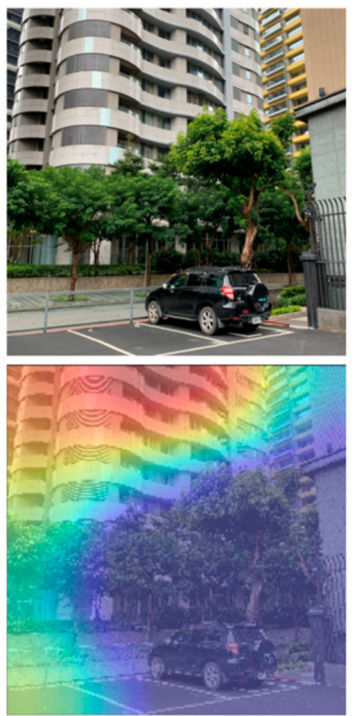

Figure 1. Grad-CAM visualizations. The top row is the original images in our tree species dataset. The bottom row is the Grad-CAM visualizations of our previous trained model. The heat maps localize class-discriminative regions, and red regions correspond to high score for class.
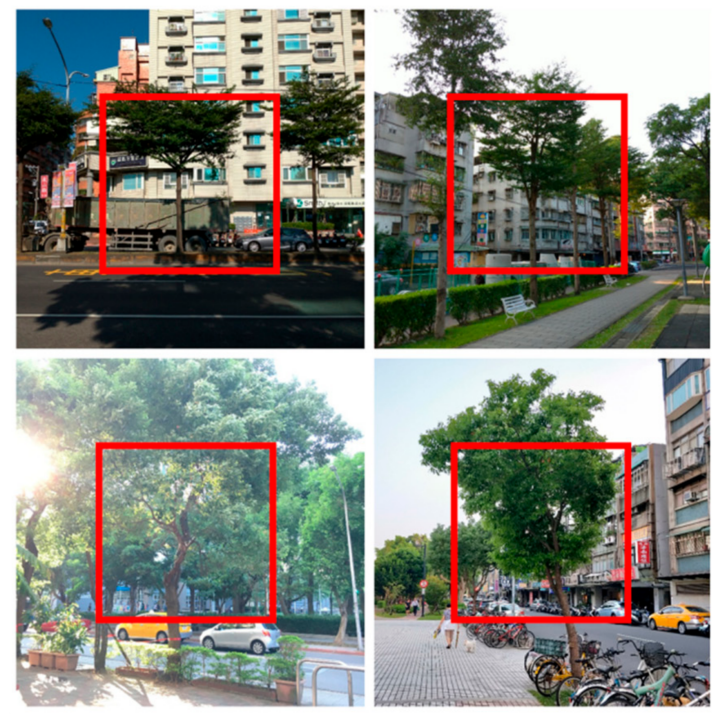
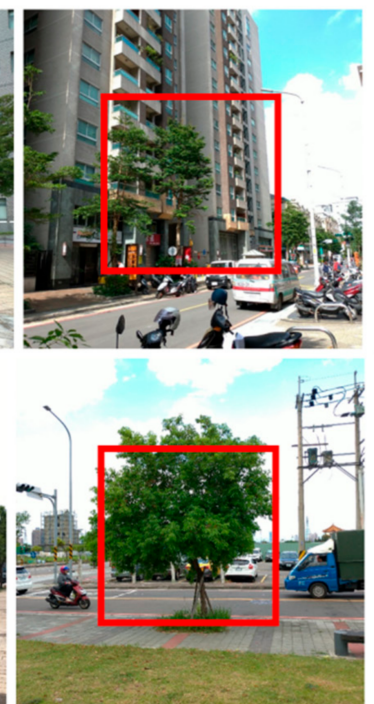

Figure 2. Samples of interfering backgrounds in the training dataset. Images in the upper row demonstrated redundant buildings and roads while in the lower row showed cars and sky as interfering background. The red boxes are the central attention part that would be cropped in the center as the input for central attention path in the dual path CNN.

\subsection{Dual-Path CNN}

We proposed a dual-path CNN architecture for tree species recognition. The architecture consisted of two sub-network pathways: (1) central attention with an InceptionV3 based pathway; and (2) an InceptionV3-based pathway. Figure 3 shows the architecture of our proposed network. Each of the two paths was independent from one another, but merged through concatenation of results, which were thus sent for classification. Feature extraction, hence comprised the classic CNN path, along with which was introduced the central attention concept, a simply visual attention focused on the central target. 


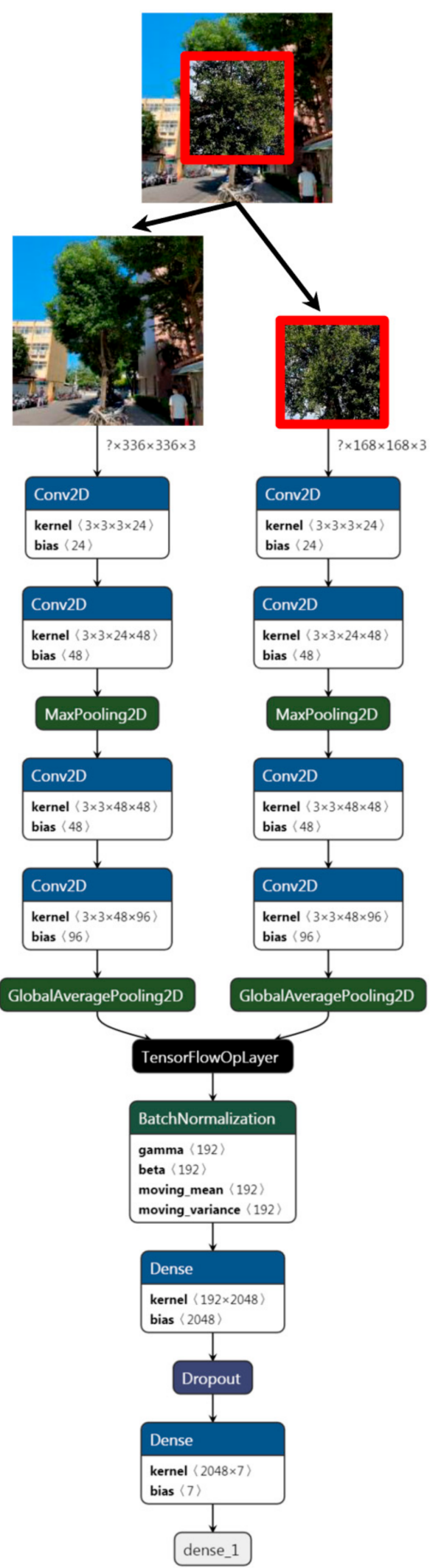

Figure 3. Architecture of the proposed dual path CNN framework. The left path is the backbone CNN architecture, and the right one is the central attention path with the central crop of the input image. Feature maps in the central attention path were concatenated with the corresponding backbone feature before class score computation. 
To find the most appropriate backbone architecture, we tested the latest CNN-based models (data not shown). Then, InceptionV3 was selected as a backbone network to solve the plant recognition problem. The InceptionV3 was pre-trained in the ImageNet1000 dataset and received a red, green, blue (RGB) image with a size of $299 \times 299$ pixels and classified into its corresponding class. Inceptionv3 introduces inception modules. Inception modules help increase the width of the network. It is a convolutional block which is constituted by different kinds of convolutional kernels. Apart from $3 \times 3$ kernel is employed which is common used, other types such as $1 \times 7,7 \times 7,1 \times 1,1 \times 3$, and so on are also adopted for constructing networks. Large and small convolutional kernels are used together in one block. Big convolutional styles and feature maps are with little number of kernels, and small convolutional styles and feature maps are with large number of kernels [19]. This architecture consists of 159 layers including an alternating sequence of convolution (CONV), pooling layers and ends with a fully connected layer [85]. This architecture includes 23 million parameters. We used this model as a feature extractor to tree classification.

Additionally, the rectified linear unit, ReLu was used for the activation of each layer except for the last. A default learning rate of 0.01 was set. The Adam optimization function was used, while the categorical cross entropy was deployed as the loss function. We trained the data for 600 epochs and set the batch size to 50 . We concatenated the results using the early fusion that performed better while integrating both networks and jointly training them end-to-end with fused representation linked directly to the species classes via a softmax layer that can be carried out before class score computation, such as during the feature learning stage, from which we adopted this method as described [9]. Afterwards, tuning the parameters of the network including activation function, mini batch size, epochs number, as well as the initial learning rate and study the accuracy and performance rate of different parameter variations of the $\mathrm{CNN}$ [86].

\subsection{Object Detection}

We employed YOLOv3 as the real-time object detection models. YOLOv3 introduced the multiscale windows generation to produce more accurate result and increased its speed substituting the backbone network with the Darknet-53 network, initialized from ImageNet [87].

The YOLOv3 model used in this work obtained the storage location of the training image and the marked pixel location of the target in the image and the class of the target by reading the txt text. This txt file was generated by reading a dataset using the VOC format. Therefore, the pictures collected in the scene were made into a dataset in the form of VOC by using an image annotation software called LabelImg [88]. It saves the object class information and position information marked in the image as a file in $\mathrm{xml}$ format for training. After using the rectangle to mark the target and select the target class, we save the file, and then the software generated the $\mathrm{xml}$ format label text with the same name as the image [89].

We also used intersection over union (IOU) and non-maximum suppression (NMS). In the training stage, we used the YOLOv3 provided code and default configuration [90,91]. The Adam optimization function was used. The learning rate was set to 0.00001 . The value for both height and width was set to $416 \times 416$. The batch size is set to 3 . Concerning the YOLOv3 parameters, the input image is subdivided to 8, 16, or 36 grids. Anchors that overlapped the ground truth object by less than a threshold value 0.5 were ignored (Figure 4). 


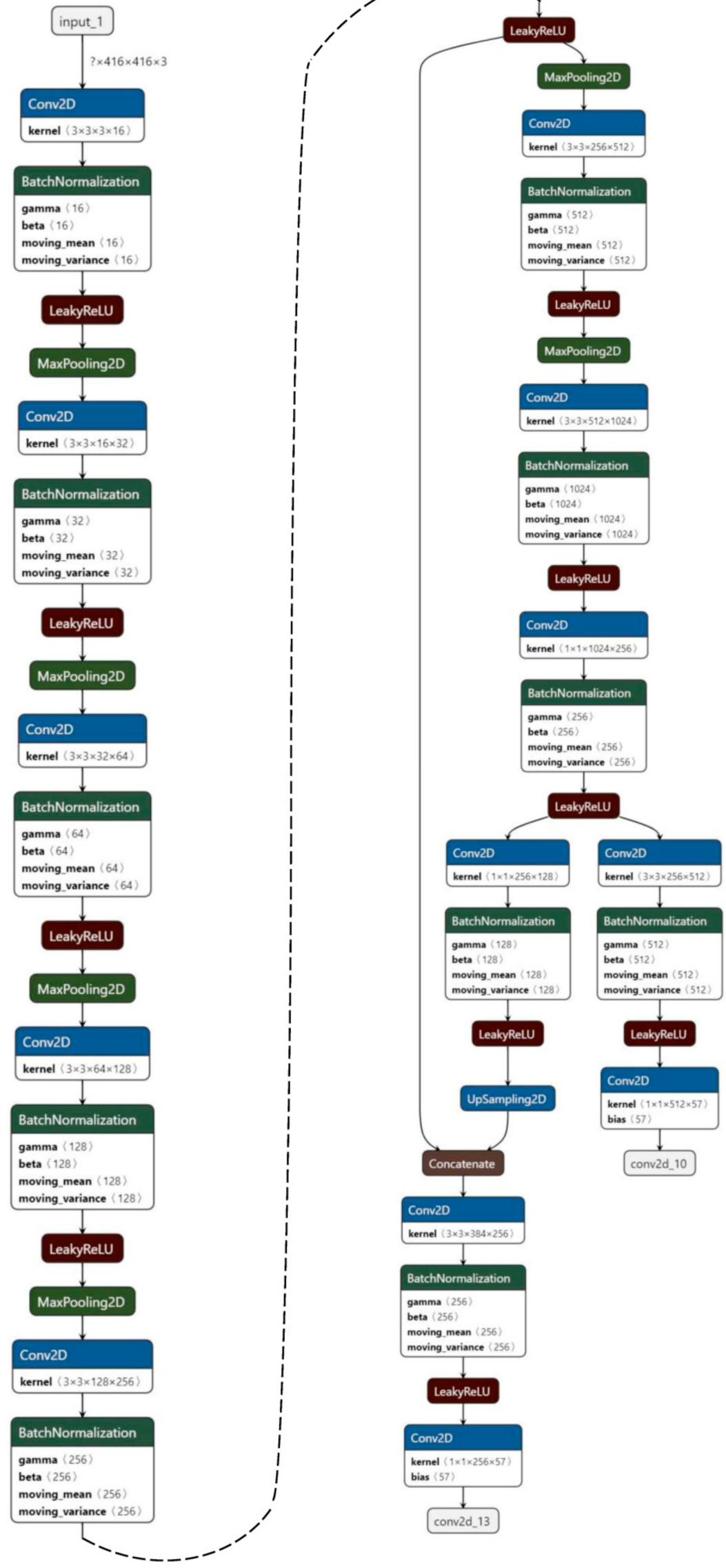

Figure 4. Object detection process flow diagram. 


\subsection{Mobile Implementation}

In our implementation, we provide interfaces to both an online and offline tree image database. The feature extraction could alternatively be performed at the server for more computationally expensive algorithms [40].

Here, we used the Tensorflow Lite framework to use in mobile environment. On Android, Tensorflow Lite leverages the Neural Networks API (NNAPI) to utilize all of the device hardware acceleration [92]. After the training was finished from a desktop environment, a Keras HDF5 model was built and then converted into a TensorFlow lite model to be embedded into the mobile environment for recognition. Based on the hardware capabilities of the device, it will efficiently distribute the workload of classification across available hardware, such as neural network hardware, GPUs, and digital signal processors (DSPs) [93].

In addition, according to the TensorFlow Lite documentation, although model quantization can lead to up to $0.8 \%$ decrease in accuracy, it may reduce the model size by four times (95.7 MB down to $23.9 \mathrm{MB}$ ) and the latency by $285 \mathrm{~ms}$ ( $1130 \mathrm{~ms}$ down to 845 $\mathrm{ms}$ ) [94]. With the optimized storage size for InceptionV3 weights, this is well-suited for mobile applications.

\section{Result}

The results demonstrate two key points. First, we build a tree species recognition system using a dual path CNN model constituted of a classic InceptionV3 CNN in combination with a central attention $\mathrm{CNN}$. This method performs better than other existing methods in the same database. Second, we deliver this identification system for Android serving not only image recognition, but also real-time object detection functions (Figure 5).

\section{Central Attention and Dual-Path CNN}

In order to evaluate the validity of the proposed framework, we use a couple of unipath $\mathrm{CNN}$ networks to conduct the same experiment and compare the experimental results. The performance is measured by the average classification accuracy of different classes. The identification accuracy of the proposed dual path CNN is $77.1 \%$ while the uni-path classic InceptionV3 CNN is $74.1 \%$ and the uni-path central attention method is $75 \%$.

In addition, we have compared the proposed CNN method with InceptionResNetV2, which has been shown to have better learning performance than InceptionV3 [95], achieve at least $99.9 \%$ accuracy on the Middle European Woods dataset [96], and also win the LifeCLEF2018 challenge [97]. The experimental results were listed in Table 4. Values for the area under the receiver operating characteristic (ROC) curves (AUC), as well as true positive rate (TPR) in each class, are presented in Table 5. As shown in Tables 4 and 5, most of the results in the proposed method for plant identification performed better than InceptionResNetV2. The possible reason may be that the amount of data collected in this study was scarce, so InceptionResNetV2, which has a more complex neural network structure, has a problem of overfitting. However, the proposed method obtains an average of $75.48 \%$ classification accuracy better than InceptionResNetV2 in the case of insufficient data, as shown in Table 4. Moreover, we used five-fold and 10-fold cross-validation to verify the effectiveness of the proposed method. Five-fold cross-validation represents that $80 \%$ of the original data is used as training data and $20 \%$ as test data. ROC curves of the top three major classes were shown in Figure 6.

Table 4. Comparison of accuracy between the proposed method and InceptionResNetV2.

\begin{tabular}{ccccc}
\hline \multirow{2}{*}{ K-Fold } & \multicolumn{2}{c}{$\begin{array}{c}\text { 5-Fold (Training Data Size = 1754, Testing Data } \\
\text { Size = 439) }\end{array}$} & $\begin{array}{c}\text { 10-Fold (Training Data Size = 1973, Testing Data } \\
\text { Size = 220) }\end{array}$ \\
\hline Method & InceptionResNetV2 & The Proposed Method & InceptionResNetV2 & The Proposed Method \\
\hline Accuracy & 56.95 & 69.02 & 71.36 & 75.48 \\
\hline
\end{tabular}




\section{A. The original Chinese interface}

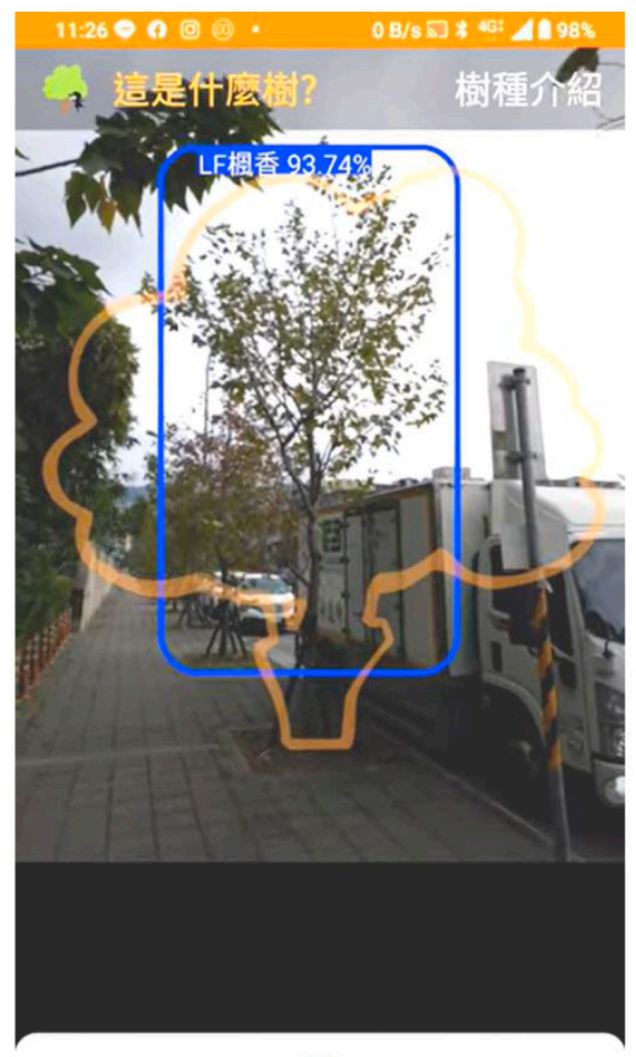

推断:

辫别方式:

解測数型。
(2) 标形
○物件偵涀

\begin{abstract}
菻形
\end{abstract}
物件分䫏

$199 \mathrm{~ms}$

\section{B. Translated in English}
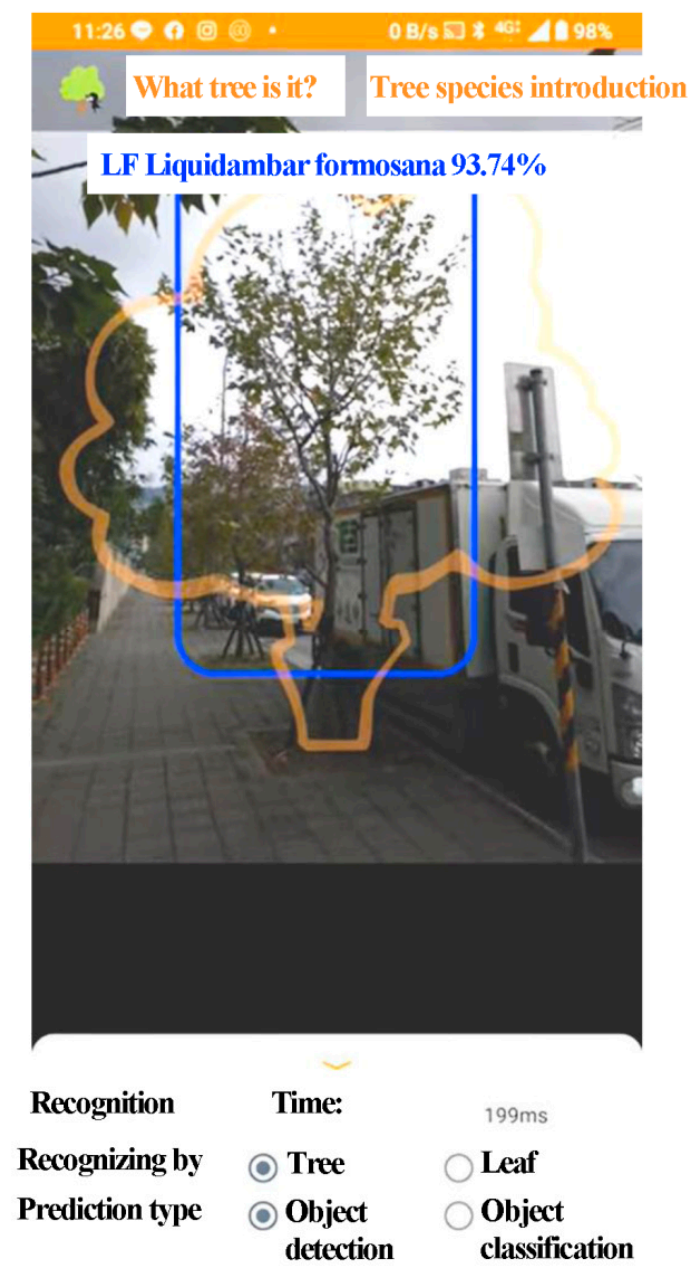

Figure 5. The screenshot of the developed mobile app. Users were instructed to place the object (tree) to be detected in the orange tree frame. In this detection, it took 199 mini seconds to frame the detected tree and show the result of Liquidambar formosana (LF). The user interface is shown in the original Chinese version (A), and translated in English (B).

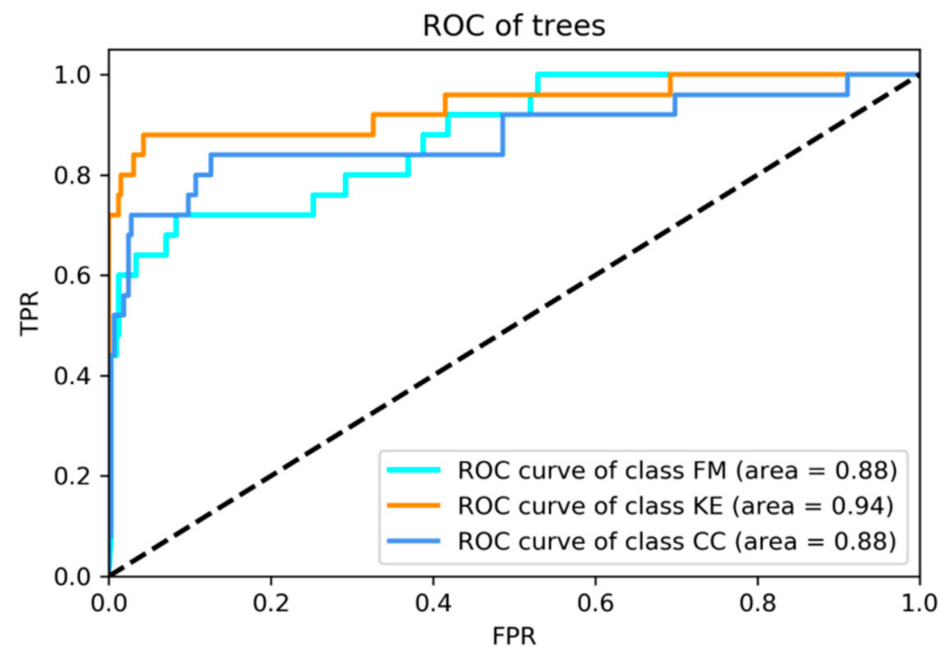

Figure 6. Receiver operating characteristic (ROC) curves of the top three major classes, Ficus microcarpa (FM), Koelreuteria henryi (KE), and Cinnamomum camphora (CC). TPR: true positive rate; FPR: false positive rate. 
Table 5. Comparison of true positive rate (TPR) and area under the curve (AUC) between the proposed method and InceptionResNetV2 in each class.

\begin{tabular}{ccccccccc}
\hline K-Fold & \multicolumn{3}{c}{ 5-Fold $\begin{array}{c}\text { (Training Data Size = 1754, Testing } \\
\text { Data Size = 439) }\end{array}$} & \multicolumn{3}{c}{$\begin{array}{c}\text { 10-Fold } \\
\text { (Training Data Size = 1843, Testing } \\
\text { Data Size = 350) }\end{array}$} \\
\hline Method & \multicolumn{3}{c}{ InceptionResNetV2 } & \multicolumn{3}{c}{$\begin{array}{c}\text { The Proposed } \\
\text { Method }\end{array}$} & InceptionResNetV2 & $\begin{array}{c}\text { The Proposed } \\
\text { Method }\end{array}$ \\
\hline Class & TPR & AUC & TPR & AUC & TPR & AUC & TPR & AUC \\
AS & 0.7297 & 0.8201 & 0.8919 & 0.8726 & 0.7500 & 0.8275 & 0.7500 & 0.8325 \\
BJ & 0.7083 & 0.7813 & 0.6875 & 0.7760 & 0.7500 & 0.8112 & 0.6250 & 0.7513 \\
CC & 0.4500 & 0.6962 & 0.4250 & 0.6849 & 0.4615 & 0.6870 & 0.6154 & 0.7561 \\
DR & 0.6667 & 0.8020 & 0.6667 & 0.8092 & 0.2500 & 0.6178 & 0.4167 & 0.6867 \\
FM & 0.7302 & 0.7959 & 0.7302 & 0.8012 & 0.7857 & 0.8304 & 0.8571 & 0.8478 \\
KE & 0.6905 & 0.7986 & 0.7857 & 0.8412 & 0.8125 & 0.8523 & 0.8125 & 0.8695 \\
LF & 0.6061 & 0.7735 & 0.7576 & 0.8320 & 0.9231 & 0.9108 & 1.0000 & 0.9517 \\
MA & 0.6316 & 0.7515 & 0.1579 & 0.5730 & 0.5000 & 0.7360 & 0.1667 & 0.5740 \\
MI & 0.1923 & 0.5877 & 0.4231 & 0.6982 & 1.0000 & 0.9328 & 0.7368 & 0.8311 \\
MP & 0.5333 & 0.7478 & 0.6000 & 0.7800 & 0.8000 & 0.8762 & 0.7000 & 0.8262 \\
PC & 0.8148 & 0.8613 & 0.8889 & 0.9080 & 1.0000 & 0.9539 & 0.9286 & 0.9182 \\
RR & 1.0000 & 0.9752 & 0.8750 & 0.9210 & 0.7500 & 0.8608 & 1.0000 & 0.9764 \\
TC & 0.3571 & 0.6715 & 0.9286 & 0.9431 & 0.7500 & 0.8608 & 1.0000 & 0.9764 \\
TM & 0.8286 & 0.8623 & 0.9143 & 0.8792 & 0.8125 & 0.8695 & 0.9375 & 0.9295 \\
Average & 0.6385 & 0.7804 & 0.6952 & 0.8085 & 0.7390 & 0.8305 & 0.7533 & 0.8377 \\
\hline
\end{tabular}

Note: Alstonia scholaris (AS), Bischofia javanica (BJ), Cinnamomum camphora (CC), Delonix regia (DR), Ficus microcarpa (FM), Koelreuteria henryi (KE), Liquidambar formosana (LF), Melia azedarach (MA), Melaleuca leucadendra (MI), Millettia pinnata (MP), Cassia fistula (PC), Roystonea regia (RR), Terminalia catappa (TC), Terminalia mantaly (TM).

The T-distributed Stochastic Neighbor Embedding ( $\mathrm{t}-\mathrm{SNE}$ ) visualization method was used to project the generated multidimensional clusters into a two-dimensional graph. This methodology is capable of maintaining the cluster distances that are present in the high dimensional space when these are projected into the two-dimensional space, and thus, the differences among clusters can be visualized [98]. Figure 7 depicted the clusters generated by each class of the 14 tree species.

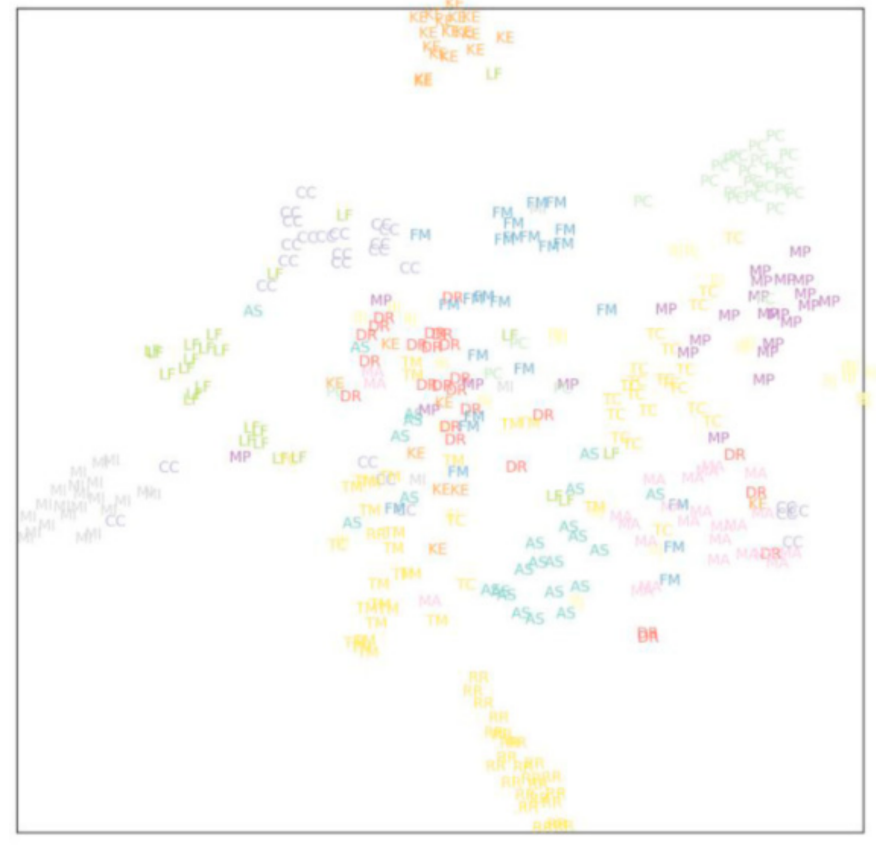

(A) presented with tree species abbreviation

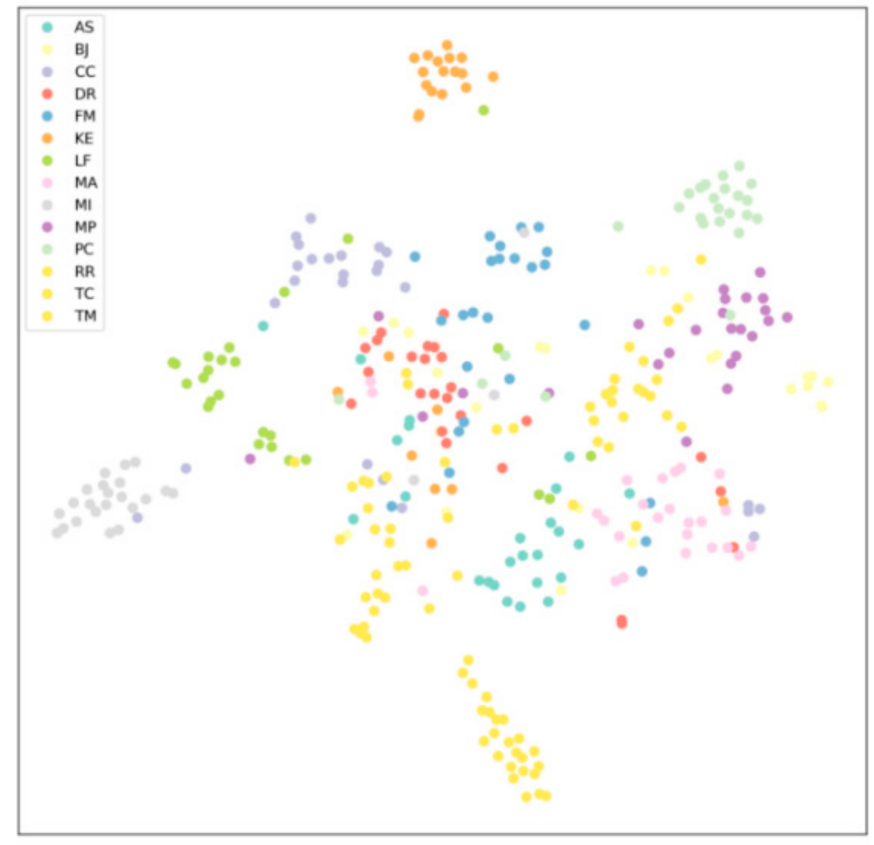

(B) presented with color dots

Figure 7. Two-dimensional T-distributed Stochastic Neighbor Embedding (T-SNE) visualization in each class of the 14 tree species is presented with tree species abbreviation (A) and with color dots (B). Alstonia scholaris (AS), Bischofia javanica (BJ), Cinnamomum camphora (CC), Delonix regia (DR), Ficus microcarpa (FM), Koelreuteria henryi (KE), Liquidambar formosana (LF), Melia azedarach (MA), Melaleuca leucadendra (MI), Millettia pinnata (MP), Cassia fistula (PC), Roystonea regia (RR), Terminalia catappa (TC), and Terminalia mantaly (TM). 
The training loss and accuracy curves of the proposed dual CNN method with epoch $=600$ and five-fold cross-validation were shown in Figure 8.

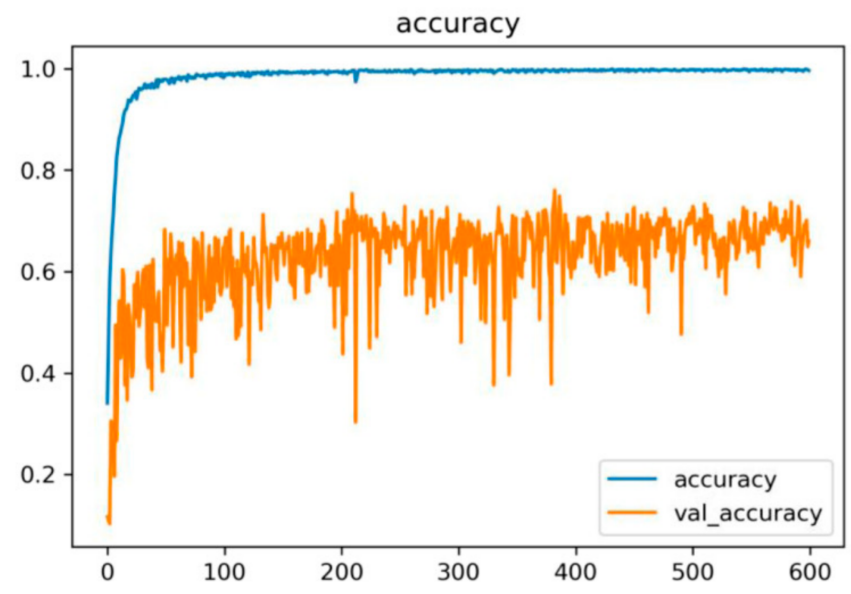

(A) accuracy and val_accuarcy

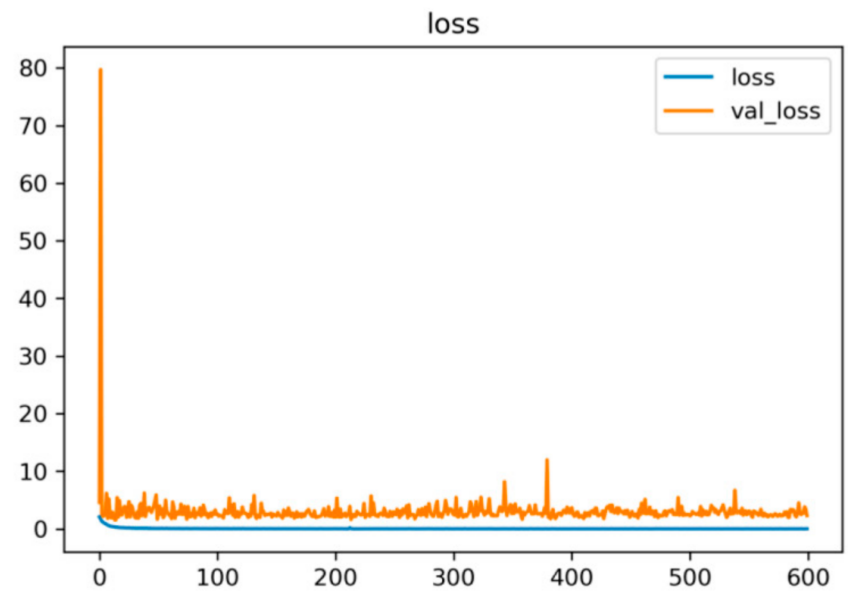

(B) loss and val_loss

Figure 8. Training accuracy (A) and loss (B) curves of the proposed dual CNN method with epoch $=600$ and five-fold cross-validation.

The Grad-CAM results of the proposed method are shown in Figure 9.
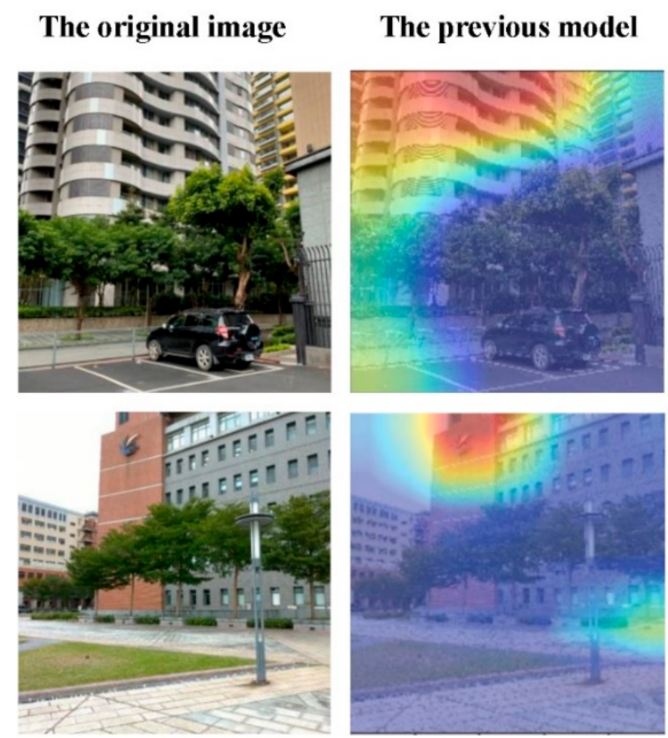

The proposed model

Figure 9. Grad-CAM visualizations of the proposed model. The left column is the original images in our tree species dataset. The middle column is the Grad-CAM visualizations of our previous trained model. The right column is the Grad-CAM results of our proposed model. The heat maps localize class-discriminative regions, and red regions correspond to high score for class.

\section{Discussion}

\subsection{Central Attention and Dual-Path CNN}

Although there are some tools already exist for plant recognition using a single organ (especially a leaf) with accuracies of $90 \%$ or more, we use the whole plant images to enable the remote recognition, which was one of the significances of this study. Moreover, the previous study showed that a plant classification method using a combination of leaf, flower and bark photos of the same tree species may achieve recognition accuracy on multi-organ better than the accuracy on a single organ [17]. In addition, previous studies 
reported 31.6\% accuracy in the LifeCLEF2019 challenge [15], 69\% top-5 identification rate in the P1@ntNet application [4], and 67\% accuracy in the iNaturalist application [44], which include the whole plant (long-range) images. In this study, the result of identification accuracy is $77.1 \%$ (Table 6), and has better learning performance (Figure 9).

Table 6. Comparison of previous dual-path CNN works and our proposed method.

\begin{tabular}{|c|c|c|c|c|}
\hline Authors, Year & Image Type & Dataset & Method & Accuracy \\
\hline $\begin{array}{l}\text { Shah et al., } \\
2017 \text { [99] }\end{array}$ & Leaf & $\begin{array}{l}\text { Flavia, Leafsnap, } \\
\text { ImageClef }\end{array}$ & $\begin{array}{l}\text { Dual-path deep convolutional } \\
\text { neural network }(\mathrm{CNN}) \\
\text { Path 1: marginalized } \\
\text { shape context } \\
\text { Path 2: shape + texture }\end{array}$ & $\begin{array}{l}\text { Flavia }(99.28 \%), \text { Leafsnap } \\
(95.61 \%), \text { ImageClef } \\
(96.42 \%)\end{array}$ \\
\hline $\begin{array}{l}\text { Lee et al., } \\
2017 \text { [9] }\end{array}$ & Leaf & $\begin{array}{l}\text { MalayaKew } \\
\text { Leaf dataset }\end{array}$ & $\begin{array}{l}\text { Dual-path CNN } \\
\text { Path 1: CNN } \\
\text { Path 2: AlexNet }\end{array}$ & $99.7 \%$ \\
\hline Rizk, 2019 [33] & Leaf & Flavia & $\begin{array}{l}\text { Dual-path CNN } \\
\text { Path 1: leaf shape } \\
\text { feature extraction } \\
\text { Path 2: leaf venation } \\
\text { feature extraction }\end{array}$ & $96.8 \%$ \\
\hline $\begin{array}{l}\text { Pan et al., } \\
2019 \text { [34] }\end{array}$ & $\begin{array}{l}\text { Pascal VOC: person, } \\
\text { animal, vehicle, indoor } \\
\text { ( } 20 \text { object classes) } \\
\text { MS COCO: animal, } \\
\text { vehicle, furniture, etc. } \\
\text { (91 classes) }\end{array}$ & $\begin{array}{l}\text { Pascal VOC and MS } \\
\text { COCO dataset }\end{array}$ & $\begin{array}{l}\text { Dual-resolution/input dual-path } \\
\text { CNNs (DualNets) } \\
\text { Path 1: backbone path } \\
\text { MobileNetV2 with } \\
300 \text { pixels input } \\
\text { Path 2: auxiliary path with larger } \\
\text { inputs ( } 600 \text { pixels) but less } \\
\text { stacked layers } \\
\text { Feature maps in the auxiliary } \\
\text { path are fused with the } \\
\text { corresponding backbone feature } \\
\text { in a residual-learning manner as } \\
\text { long as their dimensions meet. }\end{array}$ & $70.4 \%$ \\
\hline $\begin{array}{l}\text { Li et al., } \\
2018 \text { [35] }\end{array}$ & $\begin{array}{l}\text { Fire, heads, chess, } \\
\text { pumpkin, office, } \\
\text { redktichen, and stairs }\end{array}$ & $\begin{array}{l}\text { Microsoft 7-Scenes } \\
\text { dataset }\end{array}$ & $\begin{array}{l}\text { Dual-path CNN } \\
\text { Path 1: color image inputs } \\
\text { Path 2: depth image input }\end{array}$ & $\begin{array}{l}\text { Relocalization accuracy } \\
\text { improved by about } 20 \% \\
\text { compared with the } \\
\text { state-of-the-art deep } \\
\text { learning method for } \\
\text { pose regression }\end{array}$ \\
\hline $\begin{array}{l}\text { Chen and } \\
\text { Chiang, } \\
2018 \text { [37] }\end{array}$ & $\begin{array}{l}\text { Human actions } \\
\text { People Playing Musical } \\
\text { Instrument (PPMI): } \\
\text { human interaction, } \\
\text { musical instruments } \\
\text { (48 classes) } \\
\text { Willow: action ( } 7 \text { classes) } \\
\text { Uiuc-sports: action } \\
\text { (8 classes) }\end{array}$ & $\begin{array}{l}\text { PPMI dataset, } \\
\text { Willow dataset, } \\
\text { Uiuc-sports dataset }\end{array}$ & $\begin{array}{l}\text { Dual-path CNN } \\
\text { Path 1: CNN } \\
\text { Path 2: CNN pre-trained on a } \\
\text { large dataset, a large generative } \\
\text { deep learning model (e.g., } \\
\text { AlexNet, GoogleNet) }\end{array}$ & $\begin{array}{l}\text { PPMI }(42.7 \%), \text { Willow } \\
(44.8 \%), \text { Uiuc-sports } \\
(81.6 \%)\end{array}$ \\
\hline $\begin{array}{l}\text { Sun et al., } \\
2019 \text { [100] }\end{array}$ & $\begin{array}{l}\text { Car, person, bike, curve, } \\
\text { car stop, guardrail, color } \\
\text { cone, bump }\end{array}$ & $\begin{array}{l}\text { A public } \\
\text { dataset [101] }\end{array}$ & $\begin{array}{l}\text { Encoder-Decoder design } \\
\text { concept ResNet } \\
\text { RTFNet: RGB-Thermal Fusion } \\
\text { Network. Fusing both the RGB } \\
\text { and thermal information in a } \\
\text { novel deep neural network }\end{array}$ & $63.1 \%$ \\
\hline
\end{tabular}


Table 6. Cont.

\begin{tabular}{|c|c|c|c|c|}
\hline Authors, Year & Image Type & Dataset & Method & Accuracy \\
\hline $\begin{array}{l}\text { Sun et al., } \\
2020 \text { [102] }\end{array}$ & $\begin{array}{l}\text { Car, person, bike, curve, } \\
\text { car stop, guardrail, color } \\
\text { cone, bump } \\
\text { SUN-RGBD v1: room } \\
\text { scenes, furniture, etc. }\end{array}$ & $\begin{array}{l}\text { A public } \\
\text { dataset [101], } \\
\text { SUN-RGBD v1 } \\
\text { dataset [103] }\end{array}$ & $\begin{array}{l}\text { FuseSeg: RGB and thermal data } \\
\text { fusion, generally consisting of } \\
\text { two encoders to extract features } \\
\text { from input images and one } \\
\text { decoder to restore the resolution. } \\
\text { The two encoders take as input } \\
\text { the three-channel RGB and } \\
\text { one-channel thermal } \\
\text { images, respectively. } \\
\text { DenseNet-161 }\end{array}$ & $\begin{array}{l}\text { A public dataset }(70.6 \%) \\
\text { SUN-RGBD v1 }(38.3 \%)\end{array}$ \\
\hline $\begin{array}{l}\text { Wang et al., } \\
2019 \text { [104] }\end{array}$ & $\begin{array}{l}30 \text { common scenes where } \\
\text { robotic wheelchairs } \\
\text { usually work (e.g., } \\
\text { sidewalks and squares) } \\
18 \text { different kinds of road } \\
\text { anomalies that robotic } \\
\text { wheelchairs may } \\
\text { encounter in real } \\
\text { environments }\end{array}$ & $\begin{array}{l}\text { Ground mobile } \\
\text { robots perception } \\
\text { dataset (RGB-D } \\
\text { dataset) }\end{array}$ & $\begin{array}{l}\text { Self-Supervised Label Generator } \\
\text { (SSLG) + RGB-D data-based } \\
\text { semantic segmentation } \\
\text { neural networks }\end{array}$ & $75.57 \sim 88.19 \%$ \\
\hline $\begin{array}{l}\text { Our proposed } \\
\text { method }\end{array}$ & Whole plant (long-range) & $\begin{array}{l}\text { Self-collected } \\
\text { dataset: } 14 \text { species } \\
\text { of the most } \\
\text { common and } \\
\text { endemic trees } \\
\text { in Taiwan }\end{array}$ & $\begin{array}{l}\text { Dual-path CNN } \\
\text { Path 1: InceptionV3 with } \\
\text { original image inputs } \\
\text { Path 2: InceptionV3 with central } \\
\text { cropped image inputs }\end{array}$ & $77.1 \%$ \\
\hline
\end{tabular}

By combining the two networks into a unified framework, the performance of tree species recognition can be improved. The finding is in line with previous studies discussed as follows. Shah et al. used a dual-path deep CNN to learn joint feature representations for leaf images, and optimize these features for the classification task based on marginalized shape context and shape-texture dual-path deep CNN [99]. The study showed that the dual-path CNN method outperformed other CNN methods, such as uni-patch CNN, texture-patch CNN, marginalized shape context with SVM classifier, multiscale distance matrix with SVM classifier, curvature histogram on Flavia, and other datasets [105].

Lee et al. further proposed a two stream convolutional neural network (TwoCNN) [9]. In TwoCNN, the two feature learning streams were trained on the whole and patch of images respectively. Although, TwoCNN can take discriminative information at different scales (both the whole and the patches of images), the training process required a more complex sample set that must provide both whole and segmented images for this network [106].

Rizk proposed a dual path, dual feature model for plant leaf identification [33]. The author used Sobel operators as primary and secondary vein extraction for vein patch generation, and then the dual-path CNN was employed for feature extraction. The first path was for leaf shape feature extraction while the second was for leaf venation feature extraction. The results showed an accuracy of $96.8 \%$ tested on the Flavia dataset.

Dual-resolution dual-path CNNs, DualNets, were aimed to increase the object detection accuracy of small CNN models [34]. DualNets explicitly accepted dual inputs in different resolutions and extracted complementary visual features from these using dual CNN paths. The two paths in a DualNet were a backbone path (MobileNetV2) and an auxiliary path that accepted larger inputs and then rapidly down-sampled them to relatively small feature maps. Auxiliary features were extracted from the larger input with controllable computation, and then fused with the backbone features via a proposed progressive residual fusion strategy to enrich feature representation. This architecture, as the feature ex- 
tractor, was further integrated with the Single Shot Detector to accomplish latency-sensitive visual object-detection tasks. The authors evaluated the resulting detection pipeline on Pascal VOC and MS COCO benchmarks. Results showed that the DualNets can improve the accuracy of those $\mathrm{CNN}$ detection applications sensitive to computation payloads.

A proposed dual-stream CNN, designed to perform robust indoor relocalization in challenging environments, took color images and depth images as the network inputs separately [35]. The network improved the relocalization accuracy by about $20 \%$ compared to the state-of-the-art deep learning method for pose regression, and also enhanced the system robustness in challenging scenes such as large-scale, dynamic, fast movement, and night-time environments.

Chen and Chiang suggested an auxiliary structure for deep model learning with insufficient data through additional alignment layers to transfer the weight of the auxiliary model to a new one [37]. Their results demonstrated that the auxiliary structure eliminated the overfitting problem and can improve the accuracy with only a few training samples. With the replacement of different auxiliary architecture, this method can be applied in different tasks, such like detection and retrieval.

Sun et al. [100] proposed the RTFNet utilizing RGB-Thermal fusion network for semantic segmentation of urban scenes for autonomous vehicle system. They used thermal images, and fuse both the RGB and thermal information in a novel deep neural network. The encoder-decoder design concept and ResNet were employed for feature extraction. A new decoder was developed to restore the feature map resolution. Their network achieved $63.1 \%$ accuracy in a public dataset [101]. Recently, they upgraded their model by employing DenseNet-161 as the backbone of the encoders. It generally consists of two encoders to extract features from input images and one decoder to restore the resolution. The two encoders take as input the three-channel RGB and one-channel thermal images, respectively. This upgraded model, FuseSeg-161, reached $70.6 \%$ accuracy in a public dataset [101], and 38.3\% accuracy in the a RGB-Depth Scene Understanding Benchmark Suite (SUN-RGBD) v1 dataset [103], which outperformed the state of the art [102].

Wang et al. proposed a self-supervised approach to segment drivable areas and road anomalies for robotic wheelchairs. They firstly developed a pipeline named self-supervised label generator (SSLG) to automatically label drivable areas and road anomalies. Then, the segmentation labels generated by the SSLG were used to train several RGB-D databased semantic segmentation neural networks. The accuracy was $75.57 \%$ in drivable area segmentation, and $88.19 \%$ in road anomaly segmentation of their self-constructed RGB-D dataset, which covers 30 common scenes where robotic wheelchairs usually work [104].

\subsection{Challenges of Sampling in Current Automated Plant Species Identification}

Inadequate benchmark database: Large-scale, well-annotated training datasets with representative data distribution characteristics are essential for the training of accurate and generalizable classifiers in Deep CNN [4]. Entire plant instead of individual organ: Multi-organ datasets performed remarkably well with an accuracy of $100 \%$ even if the size of the dataset was small [45]. Large intraspecific and small interspecific visual variation: Even professional botanists are challenged to properly distinguish species that can be identified only by almost invisible characteristics. Images of the same organ acquired from different perspectives often contain complementary visual information that could improve accuracy in observation-based identification using multiple images [4].

Therefore, we decided to collect our own database on the entire plant, and we have been very strict to select images to establish our dataset according to the principle of high variability. The original database contains more than 30,000 photos. After deleting unqualified pictures with redundant, interfering background and non-target, it turned out just 2000 more photos finally survived. 


\subsection{Solutions to Insufficient Data Quantity}

To overcome data imbalance and overfitting, over-sampling or under-sampling can be used to adjust the class distribution from the dataset. Another simple way is to generate synthetic samples using algorithms like the Synthetic Minority Over-Sampling Technique by randomly sample the attributes from instances in the minority class. On the other hand, data augmentation can also help the network memorize the exact details of the training images via its options of resize, rotate and reflect images of the dataset [86].

In addition to increasing the data quantity by data augmentation, we proposed to improve data quality by focusing on the central attention to diminish distracting backgrounds in the natural environment. Specifically, we create an independent path with central attention images as inputs for deep learning, and finally fusion it with the other classic $\mathrm{CNN}$ path to complement the traditional deep learning network.

\subsection{Transfer Learning}

Transfer learning was adopted because it is much faster and easier to fine-tune a network than to train from scratch. Additionally, the pre-trained network has already learned a rich set of features which may be applied to a wider range of tasks [86].

Wick and Puppe proposed a pre-trained weight on the Caltech-256 dataset [107] that differed substantially from leaf classifications, and a nine-layer CNN, by which almost perfect accuracy were achieved on the Flavia and Foliage dataset [108]. Reyes et al. pretrained the proposed model with seven layers $\mathrm{CNN}$ and additional prediction layer added at the top using the ILSVRC2012 dataset of 1.8 million images, including branch, flower, fruit, leaf, stem, etc., as well as scans [109]. They used a fine-tuning end-to-end strategy without hand-engineered components to transfer learned recognition capabilities from general domains, and implemented this model on the pl@ntView dataset. The results showed that the model can classify images of flowers and leaf scans with higher accuracy than the rest of views. The model obtained an average precision of $48.6 \%$ when identifying single images in the test set. Toma et al. studied a transfer learning approach on the PlantCLEF2017 challenge for automatic plant image classification in order to evaluate the performance of a system built with noisy data against one built using trusted data. The proposed method was based on the AlexNet CNN model, and fine-tuned using PlantCLEF Encyclopedia of Life training data including 10,000 species with about 260,000 plant images. They achieved $47.03 \%$ accuracy, and ranked within top 10 in the competition [110]. Van Horn et al. used multiple pre-trained CNN models including Inception ResNet V2 Squeeze-and-Excitation (SE), Inception ResNet V2, Inception V3 SE, ResNet152 dropout layer (drp), ResNet101 drp, ResNet152, ResNet101, and MobileNet. The best validation accuracy they obtained was $67.3 \%$ top- 1 accuracy and $87.1 \%$ top- 5 accuracy on the public dataset, iNaturalist Classification and Detection Dataset, using the Inception ResNet V2 SE model [44].

This present method allows us to use the existing model with a small amount of data and training time to achieve good results that is more useful for simple classification problems with a small dataset. Besides, the parameters of this network have been reduced that break down the training difficulty. Notably, if we update the pre-training parameters of all the layers, the identification result will improve. The benefit of fine-tuning without completely retraining the model increases efficiency, as the accuracy of new training models generally increases slowly from very low values, but fine-tuning enables to get a better result with less iteration [36]. Transfer learning is efficient by using a pre-trained deep representation as a source architecture to create a new architecture [111].

Interestingly, it can use any pre-trained networks to train the new deep model given insufficient training samples or a lack of the initial weights. Each path in the dual-path deep neural network is flexible, and we can choose the different deep models according to different tasks requirement [37].

Finally, we believe further fine-tuning of the CNN parameters could enhance the overall performance and accuracy of the results. Further experiments with the model's 
weights and layer count could achieve a higher accuracy more efficiently. In addition, enlarging the database through merging with other image banks could also improve the learning process in the $\mathrm{CNN}$ [33].

\section{Conclusions}

To begin with, we propose a central attention concept based on user experience analysis and previous studies. The concept helps focus on the target instead of backgrounds in the image, and could prevent model training from confused vision for tree species recognition. Next, a dual-path $\mathrm{CNN}$ model is developed where the two different subnetworks are independent and accept individual input of either an original image or a central cropped one, respectively. A concatenation layer is then used to fuse the output of two independent sub-networks. Furthermore, we create a plant dataset of 14 species of the most common trees in Taiwan for model training and validation, respectively.

The experimental results demonstrated that the central attention combined with a dual path CNN in a whole plant recognition can achieve very competitive performance on an accuracy rate $77.1 \%$ compared to the existing deep learning algorithm, 31.6 \% accuracy in the LifeCLEF2019 challenge [15], 69\% top-5 identification rate in the P1@ntNet application [4], and $67 \%$ accuracy in the iNaturalist application [44]. Finally, we implemented the recognition model into a prototype system of an online/offline available tree species identification working on a consumer mobile platform that can not only identify the tree species by image recognition, but also real-time detect and classify for live streaming camera.

However, there are still several issues to be addressed in the future. The image data were collected from September to December in 2019. A limitation of this study is that the query image is restricted to plant species of the common roadside trees in Taiwan during fall and winter. In addition, the provided dataset, which contains only 14 species and 2332 images, would have limited field impact. However, these Taiwan-endemic roadside trees have regional specificity (e.g., Koelreuteria henryi [112], Liquidambar formosana [113]), and are possibly absent in other existing datasets. Moreover, although many datasets with thousands of species and millions of images already exist, most of them present a single organ, such as a leaf, per image. The provided dataset in this study consists of whole plant (long-range) images, facilitating not only a wealth of information on the entire tree but remote detection.

In the future we plan to extend the dataset to include more variant classes in different seasons to cover more plant species and growing stages. We also plan on trying different pre-trained CNN models to study accuracy and performance of the models. Furthermore, we plan to transfer this proposed model of image recognition to other areas such as health promotion. It has been suggested that a heterogeneous transfer learning framework may extend the transfer learning from one image classification dataset to another [114-116]. The present tree recognition model may serve as a CNN framework to apply for the health promotion area, such as automatic detection of human postures, medicine, etc., by deep transfer learning.

Author Contributions: Conceptualization, Y.C. and C.-A.C.; Data curation, Y.C. and C.-A.C.; Formal analysis, Y.C., C.-A.C. and C.-Y.L.; Funding acquisition, Y.C.; Investigation, Y.C. and C.-A.C.; Methodology, Y.C. and C.-A.C.; Project administration, Y.C. and C.-A.C.; Resources, Y.C. and C.-A.C.; Software, Y.C., C.-A.C. and C.-Y.L.; Supervision, Y.C. and C.-A.C.; Validation, Y.C. and C.-A.C.; Visualization, Y.C. and C.-A.C.; Writing-original draft, Y.C.; Writing-review \& editing, Y.C. All authors have read and agreed to the published version of the manuscript.

Funding: This research was funded by National Taipei University of Nursing and Health Sciences (110ntunhs-NT-01).

Institutional Review Board Statement: Not applicable.

Informed Consent Statement: Not applicable.

Data Availability Statement: Data sharing is not applicable to this article. 
Acknowledgments: We would like to thank Po-Yu Lai, Chung-Rui Chao, Bing-Shi Tsai, Hsun-Kun Cheng, Wei-Chun Lai, Pu-Chung Chen, Chien-Yu Fang, Ting-Wei Su, and Yu-Hsuan Lin for technical support, and Wei-Chen Chang for administrative management. We are grateful to all the subjects of which participation made this study possible.

Conflicts of Interest: The authors declare no conflict of interest.

\section{References}

1. Bodhwani, V.; Acharjya, D.; Bodhwani, U. Deep Residual Networks for Plant Identification. Procedia Comput. Sci. 2019, 152, 186-194. [CrossRef]

2. Elphick, C.S. How you count counts: The importance of methods research in applied ecology. J. Appl. Ecol. 2008, 45, 1313-1320. [CrossRef]

3. Darwin, C.R. On the Origin of Species by Means of Natural Selection, or the Preservation of Favoured Races in the Struggle for Life. Br. Foreign Med. Chir. Rev. 1860, 25, 367-404.

4. Wäldchen, J.; Rzanny, M.; Seeland, M.; Mäder, P. Automated plant species identification—Trends and future directions. PLoS Comput. Biol. 2018, 14, e1005993. [CrossRef]

5. Ceballos, G.; Ehrlich, P.R.; Barnosky, A.D.; García, A.; Pringle, R.M.; Palmer, T.M. Accelerated modern human-induced species losses: Entering the sixth mass extinction. Sci. Adv. 2015, 1, e1400253. [CrossRef]

6. Hopkins, G.W.; Freckleton, R.P. Declines in the numbers of amateur and professional taxonomists: Implications for conservation. Anim. Conserv. 2002, 5, 245-249. [CrossRef]

7. Bertrand, S.; Ben Ameur, R.; Cerutti, G.; Coquin, D.; Valet, L.; Tougne, L. Bark and leaf fusion systems to improve automatic tree species recognition. Ecol. Inform. 2018, 46, 57-73. [CrossRef]

8. $\quad$ Austen, G.E.; Bindemann, M.; Griffiths, R.A.; Roberts, D.L. Species identification by experts and non-experts: Comparing images from field guides. Sci. Rep. 2016, 6, 33634. [CrossRef]

9. Lee, S.H.; Chan, C.S.; Mayo, S.J.; Remagnino, P. How deep learning extracts and learns leaf features for plant classification. Pattern Recognit. 2017, 71, 1-13. [CrossRef]

10. Gao, M.; Lin, L.; Sinnott, R.O. A Mobile Application for Plant Recognition through Deep Learning. In Proceedings of the 2017 IEEE 13th International Conference on e-Science (e-Science), Auckland, New Zealand, 24-27 October 2017; pp. 29-38.

11. Söderkvist, O. Computer Vision Classification of Leaves from Swedish Trees. Master's Thesis, Linköping University, Linköping, Sweden, 2001.

12. Wu, S.G.; Bao, F.S.; Xu, E.Y.; Wang, Y.-X.; Chang, Y.-F.; Xiang, Q.-L. A leaf recognition algorithm for plant classification using probabilistic neural network. In Proceedings of the 2007 IEEE international symposium on signal processing and information technology, Giza, Egypt, 15-18 December 2007; pp. 11-16.

13. Agarwal, G.; Belhumeur, P.; Feiner, S.; Jacobs, D.; Kress, W.J.; Ramamoorthi, R.; Bourg, N.A.; Dixit, N.; Ling, H.; Mahajan, D. First steps toward an electronic field guide for plants. Taxon 2006, 55, 597-610. [CrossRef]

14. Hu, R.; Jia, W.; Ling, H.; Huang, D. Multiscale distance matrix for fast plant leaf recognition. IEEE Trans. Image Process. 2012, 21, $4667-4672$

15. Goëau, H.; Bonnet, P.; Joly, A. Overview of Life CLEF Plant Identification task 2019: Diving into data deficient tropical countries. In Proceedings of the CLEF 2019-Conference and Labs of the Evaluation Forum, Lugano, Switzerland, 9 September 2019; pp. 1-13.

16. Qi, Y.; Zhang, S.; Qin, L.; Huang, Q.; Yao, H.; Lim, J.; Yang, M.-H. Hedging deep features for visual tracking. IEEE Trans. Pattern Anal. Machine Intell. 2018, 41, 1116-1130. [CrossRef]

17. Kim, S.; Kim, B.; Kim, D. Tree recognition for landscape using by combination of features of its leaf, flower and bark. In Proceedings of the SICE Annual Conference 2011, Tokyo, Japan, 13-18 September 2011; pp. 1147-1151.

18. Feitoza, M.C.; da Silva, W.B.; Calumby, R.T. Exploring Deep Features and Transfer Learning for Plant Species Recognition. In Proceedings of the XV Brazilian Symposium on Information Systems, Aracaju, Brazil, 8 May 2019; p. 54.

19. Xiao, Q.; Li, G.; Xie, L.; Chen, Q. Real-world plant species identification based on deep convolutional neural networks and visual attention. Ecol. Inform. 2018, 48, 117-124. [CrossRef]

20. Li, J.; Levine, M.D.; An, X.; Xu, X.; He, H. Visual saliency based on scale-space analysis in the frequency domain. IEEE Trans. Pattern Anal. Mach. Intell. 2012, 35, 996-1010. [CrossRef]

21. Abdelmaguid, E.; Huang, J.; Kenchareddy, S.; Singla, D.; Wilke, L.; Nguyen, M.H.; Altintas, I. Left ventricle segmentation and volume estimation on cardiac mri using deep learning. arXiv 2018, arXiv:1809.06247.

22. Vig, E.; Dorr, M.; Cox, D. Large-scale optimization of hierarchical features for saliency prediction in natural images. In Proceedings of the IEEE Conference on Computer Vision and Pattern Recognition, Columbus, OH, USA, 23-28 June 2014; pp. 2798-2805.

23. Liu, N.; Han, J.; Zhang, D.; Wen, S.; Liu, T. Predicting eye fixations using convolutional neural networks. In Proceedings of the IEEE Conference on Computer Vision and Pattern Recognition, Boston, MA, USA, 7-12 June 2015; pp. 362-370.

24. Jiang, M.; Huang, S.; Duan, J.; Zhao, Q. Salicon: Saliency in context. In Proceedings of the IEEE conference on computer vision and pattern recognition, Boston, MA, USA, 7-12 June 2015; pp. 1072-1080. 
25. Pan, J.; Sayrol, E.; Giro-i-Nieto, X.; McGuinness, K.; O'Connor, N.E. Shallow and deep convolutional networks for saliency prediction. In Proceedings of the IEEE Conference on Computer Vision and Pattern Recognition, Las Vegas, NV, USA, 27-30 June 2016; pp. 598-606.

26. Marchesotti, L.; Cifarelli, C.; Csurka, G. A framework for visual saliency detection with applications to image thumbnailing. In Proceedings of the 2009 IEEE 12th International Conference on Computer Vision, Kyoto, Japan, 29 September-2 October 2009; pp. 2232-2239.

27. Sun, J.; Ling, H. Scale and object aware image thumbnailing. Int. J. Comput. Vis. 2013, 104, 135-153. [CrossRef]

28. Chen, J.; Bai, G.; Liang, S.; Li, Z. Automatic image cropping: A computational complexity study. In Proceedings of the IEEE Conference on Computer Vision and Pattern Recognition, Las Vegas, NV, USA, 27-30 June 2016; pp. 507-515.

29. Wang, W.; Shen, J.; Porikli, F. Saliency-aware geodesic video object segmentation. In Proceedings of the IEEE conference on computer vision and pattern recognition, Boston, MA, USA, 7-12 June 2015; pp. 3395-3402.

30. Wang, W.; Shen, J.; Shao, L. Consistent Video Saliency Using Local Gradient Flow Optimization and Global Refinement. IEEE Trans. Image Process. 2015, 24, 4185-4196. [CrossRef]

31. Wang, W.; Shen, J.; Shao, L.; Porikli, F. Correspondence driven saliency transfer. IEEE Trans. Image Process. 2016, 25, 5025-5034. [CrossRef]

32. Wang, W.; Shen, J.; Yang, R.; Porikli, F. Saliency-aware video object segmentation. IEEE Trans. Pattern Anal. Machine Intell. 2017, 40, 20-33. [CrossRef]

33. Rizk, S. Plant Leaf Classification Using Dual Path Convolutional Neural Networks. Master's Thesis, Notre Dame UniversityLouaize, Kesrwan, Lebanon, 2019.

34. Pan, J.; Sun, H.; Song, Z.; Han, J. Dual-Resolution Dual-Path Convolutional Neural Networks for Fast Object Detection. Sensors 2019, 19, 3111. [CrossRef]

35. Li, R.; Liu, Q.; Gui, J.; Gu, D.; Hu, H. Indoor Relocalization in Challenging Environments With Dual-Stream Convolutional Neural Networks. IEEE Trans. Autom. Sci. Eng. 2018, 15, 651-662. [CrossRef]

36. He, G.; Xia, Z.; Zhang, Q.; Zhang, H.; Fan, J. Plant Species Identification by Bi-channel Deep Convolutional Networks. J. Phys. Conf. Ser. 2018, 1004, 012015. [CrossRef]

37. Chen, C.-Y.; Chiang, C.-K. Auxiliary Structure for Convolutional Neural Network Training. In Proceedings of the 2018 Asia-Pacific Signal and Information Processing Association Annual Summit and Conference (APSIPA ASC), Honolulu, HI, USA, 12-15 November 2018; pp. 1995-1999.

38. Weng, Y.; Zeng, R.; Wu, C.; Wang, M.; Wang, X.; Liu, Y. A survey on deep-learning-based plant phenotype research in agriculture. Sci. Sin. Vitae 2019, 49, 698-716. [CrossRef]

39. Wäldchen, J.; Mäder, P. Plant Species Identification Using Computer Vision Techniques: A Systematic Literature Review. Arch. Comput. Methods Eng. 2018, 25, 507-543. [CrossRef]

40. Wang, B.; Brown, D.; Gao, Y.; Salle, J.L. Mobile plant leaf identification using smart-phones. In Proceedings of the 2013 IEEE International Conference on Image Processing, Melbourne, Australia, 15-18 September 2013; pp. 4417-4421.

41. Glority Software Ltd. Xingse. Available online: http://www.xingseapp.com/ (accessed on 1 December 2020).

42. Kumar, N.; Belhumeur, P.N.; Biswas, A.; Jacobs, D.W.; Kress, W.J.; Lopez, I.C.; Soares, J.V.B. Leafsnap: A Computer Vision System for Automatic Plant Species Identification. In Proceedings of the European conference on computer vision, Florence, Italy, 7-13 October 2012; pp. 502-516.

43. Goëau, H.; Bonnet, P.; Joly, A.; Bakić, V.; Barbe, J.; Yahiaoui, I.; Selmi, S.; Carré, J.; Barthélémy, D.; Boujemaa, N. Pl@ ntnet mobile app. In Proceedings of the 21st ACM International Conference on Multimedia, Barcelona, Spain, 21-25 October 2013; pp. 423-424.

44. Van Horn, G.; Mac Aodha, O.; Song, Y.; Cui, Y.; Sun, C.; Shepard, A.; Adam, H.; Perona, P.; Belongie, S. The inaturalist species classification and detection dataset. In Proceedings of the IEEE Conference on Computer Vision and Pattern Recognition, Salt Lake City, UT, USA, 18-23 June 2018; pp. 8769-8778.

45. Zhu, H.; Liu, Q.; Qi, Y.; Huang, X.; Jiang, F.; Zhang, S. Plant identification based on very deep convolutional neural networks. Multimedia Tools Appl. 2018, 77, 29779-29797. [CrossRef]

46. Lee, S.H.; Chan, C.S.; Wilkin, P.; Remagnino, P. Deep-plant: Plant identification with convolutional neural networks. In Proceedings of the 2015 IEEE International Conference on Image Processing (ICIP), Quebec City, QC, Canada, 27-30 September 2015; pp. 452-456.

47. Chopra, M. TreeID: An Image Recognition System for Plant Species Identification; Report in cs231n of Stanford University: Stanford, CA, USA, 2015.

48. Wu, Y.-H.; Shang, L.; Huang, Z.-K.; Wang, G.; Zhang, X.-P. Convolutional Neural Network Application on Leaf Classification. In Proceedings of the International Conference on Intelligent Computing (ICIC): Intelligent Computing Theories and Application Cham, Lanzhou, China, 2-5 August 2016; pp. 12-17.

49. Quoc Bao, T.; Tan Kiet, N.T.; Quoc Dinh, T.; Hiep, H.X. Plant species identification from leaf patterns using histogram of oriented gradients feature space and convolution neural networks. J. Info. Telecommun. 2019, 4, 1-11. [CrossRef]

50. Zhang, C.; Zhou, P.; Li, C.; Liu, L. A convolutional neural network for leaves recognition using data augmentation. In Proceedings of the 2015 IEEE International Conference on Computer and Information Technology; Ubiquitous Computing and Communications; Dependable, Autonomic and Secure Computing; Pervasive Intelligence and Computing, Liverpool, UK, 26-28 October 2015; pp. 2143-2150. 
51. Barré, P.; Stöver, B.C.; Müller, K.; Steinhage, V. LeafNet: A computer vision system for automatic plant species identification. Ecol. Inform. 2017, 40, 50-56. [CrossRef]

52. Pearline, S.A.; Kumar, V.S.; Harini, S. A study on plant recognition using conventional image processing and deep learning approaches. J. Intell. Fuzzy Syst. 2019, 36, 1997-2004. [CrossRef]

53. Sun, Y.; Liu, Y.; Wang, G.; Zhang, H. Deep Learning for Plant Identification in Natural Environment. Comput. Intell. Neurosci. 2017, 2017, 1-6. [CrossRef]

54. Joly, A.; Goëau, H.; Botella, C.; Glotin, H.; Bonnet, P.; Vellinga, W.-P.; Planqué, R.; Müller, H. Overview of LifeCLEF 2018: A Large-Scale Evaluation of Species Identification and Recommendation Algorithms in the Era of AI; CLEF (Cross-Language Evaluation Forum): Avignon, France, 2018; pp. 247-266.

55. He, K.; Zhang, X.; Ren, S.; Sun, J. Identity Mappings in Deep Residual Networks. In Proceedings of the European Conference on Computer Vision (ECCV 2016), Amsterdam, The Netherlands, 11-14 October 2016; pp. 630-645.

56. Belhumeur, P.N.; Chen, D.; Feiner, S.; Jacobs, D.W.; Kress, W.J.; Ling, H.; Lopez, I.; Ramamoorthi, R.; Sheorey, S.; White, S.; et al. Searching the World's Herbaria: A System for Visual Identification of Plant Species. In Proceedings of the European Conference on Computer Vision (ECCV 2008), Marseille, France, 12-18 October 2008; pp. 116-129.

57. Cerutti, G.; Tougne, L.; Mille, J.; Vacavant, A.; Coquin, D. Understanding leaves in natural images-A model-based approach for tree species identification. Comput. Vis. Image Underst. 2013, 117, 1482-1501. [CrossRef]

58. Ma, L.-H.; Zhao, Z.-Q.; Wang, J. ApLeafis: An Android-Based Plant Leaf Identification System. Comput. Vis. 2013, 7995, $106-111$.

59. Nguyen, Q.-K.; Le, T.-L.; Pham, N.-H. Leaf based plant identification system for Android using SURF features in combination with Bag of Words model and supervised learning. In Proceedings of the 2013 International Conference on Advanced Technologies for Communications (ATC 2013), Ho Chi Minh City, Vietnam, 16-18 October 2013; pp. 404-407.

60. Carranza-Rojas, J.; Mata-Montero, E. Combining leaf shape and texture for Costa Rican plant species identification. CLEI Electr. J. 2016, 19, 7 .

61. Prasad, S.; Peddoju, S.K.; Ghosh, D.; Prasad, S. Mobile plant species classification: A low computational approach. In Proceedings of the 2013 IEEE Second International Conference on Image Information Processing (ICIIP-2013), Shimla, India, 9-11 December 2013; pp. 405-409.

62. Priyankara, H.A.C.; Withanage, D.K. Computer assisted plant identification system for Android. In Proceedings of the 2015 Moratuwa Engineering Research Conference (MERCon), Moratuwa, Sri Lanka, 7-8 April 2015; pp. 148-153.

63. Zhao, Z.-Q.; Ma, L.-H.; Cheung, Y.-M.; Wu, X.; Tang, Y.; Chen, C.L.P. ApLeaf: An efficient android-based plant leaf identification system. Neurocomputing 2015, 151, 1112-1119. [CrossRef]

64. Gajjar, V.; Lai, Z.-H.; Kosbar, K. Fast classification of leaf images for agricultural remote sensing applications. Int. Telemeter. Conf. Proceed. 2018, 54.

65. Mishra, P.K.; Maurya, S.K.; Singh, R.K.; Misra, A.K. A semi automatic plant identification based on digital leaf and flower images. In Proceedings of the IEEE-International Conference On Advances In Engineering, Science And Management (ICAESM-2012), Nagapattinam, India, 30-31 March 2012; pp. 68-73.

66. Columbia University; University of Maryland; Smithsonian Institution. Leafsnap. Available online: https://itunes.apple.com/ us/app/leafsnap/id430649829 (accessed on 1 December 2020).

67. Affouard, A.; Goëau, H.; Bonnet, P.; Lombardo, J.-C.; Joly, A. Pl@ ntnet app in the era of deep learning. In Proceedings of the ICLR 2017, Toulon, France, 24-26 April 2017.

68. Joly, A.; Müller, H.; Goëau, H.; Glotin, H.; Spampinato, C.; Rauber, A.; Bonnet, P.; Vellinga, W.-P.; Fisher, B. LifeCLEF: Multimedia Life Species Identification; EMR@ ICMR: Orlando, FL, USA, 2014; pp. 7-13.

69. Crocker, E.V.; Condon, B.; Almsaeed, A.; Jarret, B.; Nelson, C.D.; Abbott, A.G.; Main, D.; Staton, M.E. TreeSnap: A citizen science app connecting tree enthusiasts and forest scientists. PLANTS PEOPLE Planet. 2019, 2, 47-52. [CrossRef]

70. Ralls, E. Systems and methods for electronically identifying plant species. Google Patents WO2020056148A1, 2018.

71. Cody, C.; Brown, C.; Mignotte, N. WildSnap: Mobile Android Application for Wildlife/Plant Identification; North Carolina State University: Raleigh, NC, USA, 2018.

72. Kesifler Dunyasi LTD. Discovery Green Lab. Forest Tree Identification. Available online: https://play.google.com/store/apps/ details?id=com.kesiflerdunyasi.foresttreeidentification\&hl=en_US (accessed on 1 December 2020).

73. Rahman, M.; Khan, A.A.; Shameem, M.; Uddin, B. Flower Identification Using Machine Learning. Bachelor's Thesis, Daffodil International University, Dhaka, Bangladesh, 2018.

74. Levesquea, C. About My Woods-A New Mobile App for Forest Landowners: A New Tool to Encourage Stewardship. J. Forest. 2016, 114, 170-171.

75. Bugwood. Southeast Early Detection Network. Available online: https://apps.bugwood.org/apps/seedn/ (accessed on 1 December 2020).

76. Gee, S. iNaturalist Launches Deep Learning-Based Identification App. Available online: https://www.i-programmer.info/news/ 105-artificial-intelligence/10848-inaturalist.html/ (accessed on 1 December 2020).

77. Wäldchen, J.; Mäder, P. Machine learning for image based species identification. Methods Ecol. Evol. 2018, 9, 2216-2225. [CrossRef]

78. Goëau, H.; Joly, A.; Bonnet, P.; Selmi, S.; Molino, J.-F.; Barthélémy, D.; Boujemaa, N. Lifeclef plant identification task. In Proceedings of the CLEF2014 Working Notes. Working Notes for CLEF 2014 Conference, Sheffield, UK, 15-18 September 2014 ; pp. 598-615. 
79. Parks and Street Lights Office; Public Works Department. Street Trees Information Website. Available online: https://trees.gov. taipei (accessed on 1 December 2020).

80. Shao, K.T. Catalogue of Life in Taiwan. Available online: http:/ / taibnet.sinica.edu.tw (accessed on 1 December 2020).

81. Lu, F.Y.; Ou, C.X.; Lu, J.C. Taiwan Tree Commentary; Council of Agriculture, Executive Yuan: Taipei, Taiwan, 2000.

82. Selvaraju, R.R.; Cogswell, M.; Das, A.; Vedantam, R.; Parikh, D.; Batra, D. Grad-CAM: Visual Explanations from Deep Networks via Gradient-Based Localization. In Proceedings of the 2017 IEEE International Conference on Computer Vision (ICCV), Venice, Italy, 22-29 October 2017; pp. 618-626.

83. Abadi, M.; Agarwal, A.; Barham, P.; Brevdo, E.; Chen, Z.; Citro, C.; Corrado, G.S.; Davis, A.; Dean, J.; Devin, M. Tensorflow: Large-scale machine learning on heterogeneous distributed systems. arXiv 2016, arXiv:1603.04467.

84. Lasseck, M. Image-based Plant Species Identification with Deep Convolutional Neural Networks. In Proceedings of the CLEF (Working Notes), CLEF 2017—Conference and Labs of the Evaluation Forum, Dublin, Ireland, 11-14 September 2017.

85. Szegedy, C.; Vanhoucke, V.; Ioffe, S.; Shlens, J.; Wojna, Z. Rethinking the inception architecture for computer vision. Conf. Proc. 2016, 2818-2826. [CrossRef]

86. Al-Qurran, R.; Al-Ayyoub, M.; Shatnawi, A. Plant Classification in the Wild: A Transfer Learning Approach. In Proceedings of the 2018 International Arab Conference on Information Technology (ACIT), Werdanye, Lebanon, 28-30 November 2018; pp. 1-5.

87. Redmon, J.; Farhadi, A. Yolov3: An incremental improvement. arXiv 2018, arXiv:1804.02767.

88. Tzutalin. LabelImg. Git Code. Available online: https://github.com/tzutalin/labelImg (accessed on 1 December 2020).

89. Xiao, D.; Shan, F.; Li, Z.; Le, B.T.; Liu, X.; Li, X. A target detection model based on improved tiny-Yolov3 under the environment of mining truck. IEEE Access 2019, 7, 123757-123764. [CrossRef]

90. Anh, H.N. Training and Detecting Objects with YOLO. Available online: https://github.com/experiencor/keras-yolo3 (accessed on 1 December 2020).

91. qqwweee. A Keras Implementation of YOLOv3 (Tensorflow Backend). Available online: https://github.com/qqwweee/kerasyolo3 (accessed on 1 December 2020).

92. Chong, K.; Ibrahim, A. Bringing defensive artificial intelligence capabilities to mobile devices. In Proceedings of the 16th Australian Information Security Management Conference, Perth, Australia, 4-6 January 2018; pp. 41-50.

93. Richoz, S.; Perez-Uribe, A.; Birch, P.; Roggen, D. Benchmarking deep classifiers on mobile devices for vision-based transportation recognition. In Proceedings of the Adjunct Proceedings of the 2019 ACM International Joint Conference on Pervasive and Ubiquitous Computing and Proceedings of the 2019 ACM International Symposium on Wearable Computers, London, UK, 11-13 September 2019; Association for Computing Machinery (ACM): London, UK, 2019; pp. 803-807.

94. TensorFlow. Model Optimization. Available online: https://www.tensorflow.org/lite/performance/model_optimization (accessed on 1 December 2020).

95. Szegedy, C.; Ioffe, S.; Vanhoucke, V.; Alemi, A. Inception-v4, inception-resnet and the impact of residual connections on learning. arXiv 2016, arXiv:1602.07261.

96. Šulc, M.; Matas, J. Fine-grained recognition of plants from images. Plant. Methods 2017, 13, 1-14. [CrossRef]

97. Sulc, M.; Picek, L.; Matas, J. Plant Recognition by Inception Networks with Test-time Class Prior Estimation. In Proceedings of the CLEF (Working Notes), Avignon, France, 10-14 September 2018.

98. Picon, A.; Medela, A. Constellation loss: Improving the efficiency of deep metric learning loss functions for the optimal embedding of histopathological images. J. Pathol. Inform. 2020, 11, 38. [CrossRef]

99. Shah, M.P.; Singha, S.; Awate, S.P. Leaf classification using marginalized shape context and shape+texture dual-path deep convolutional neural network. In Proceedings of the 2017 IEEE International Conference on Image Processing (ICIP), Beijing, China, 17-20 September 2017; pp. 860-864.

100. Sun, Y.; Zuo, W.; Liu, M. RTFNet: RGB-Thermal Fusion Network for Semantic Segmentation of Urban Scenes. IEEE Robot. Autom. Lett. 2019, 4, 2576-2583. [CrossRef]

101. Ha, Q.; Watanabe, K.; Karasawa, T.; Ushiku, Y.; Harada, T. MFNet: Towards real-time semantic segmentation for autonomous vehicles with multi-spectral scenes. In Proceedings of the 2017 IEEE/RSJ International Conference on Intelligent Robots and Systems (IROS), Vancouver, BC, Canada, 24-28 September 2017; pp. 5108-5115.

102. Sun, Y.; Zuo, W.; Yun, P.; Wang, H.; Liu, M. FuseSeg: Semantic Segmentation of Urban Scenes Based on RGB and Thermal Data Fusion. IEEE Trans. Autom. Sci. Eng. 2020, 1-12. [CrossRef]

103. Song, S.; Lichtenberg, S.P.; Xiao, J. SUN RGB-D: A RGB-D scene understanding benchmark suite. In Proceedings of the 2015 IEEE Conference on Computer Vision and Pattern Recognition (CVPR), Boston, MA, USA, 7-12 June 2015; pp. 567-576.

104. Wang, H.; Sun, Y.; Liu, M. Self-Supervised Drivable Area and Road Anomaly Segmentation Using RGB-D Data For Robotic Wheelchairs. IEEE Robot. Autom. Lett. 2019, 4, 4386-4393. [CrossRef]

105. Azlah, M.A.F.; Chua, L.S.; Rahmad, F.R.; Abdullah, F.I.; Alwi, S.R.W. Review on Techniques for Plant Leaf Classification and Recognition. Computers 2019, 8, 77. [CrossRef]

106. Hu, J.; Chen, Z.; Yang, M.; Zhang, R.; Cui, Y. A Multiscale Fusion Convolutional Neural Network for Plant Leaf Recognition. IEEE Signal. Process. Lett. 2018, 25, 853-857. [CrossRef]

107. Griffin, G.; Holub, A.; Perona, P. Caltech-256 Object Category Dataset; CalTech Report: Pasadena, CA, USA, 2007.

108. Wick, C.; Puppe, F. Leaf Identification Using a Deep Convolutional Neural Network. arXiv 2017, arXiv:1712.00967. 
109. Reyes, A.K.; Caicedo, J.C.; Camargo, J.E. Fine-tuning Deep Convolutional Networks for Plant Recognition. CLEF 2015, 1391, $467-475$.

110. Toma, A.; Stefan, L.-D.; Ionescu, B. UPB HES SO@ PlantCLEF 2017: Automatic Plant Image Identification using Transfer Learning via Convolutional Neural Networks. In Proceedings of the CLEF (Working Notes), Dublin, Ireland, 11-14 September 2017.

111. Su, Y.; Chiu, T.; Yeh, C.; Huang, H.; Hsu, W.H. Transfer learning for video recognition with scarce training data. arXiv 2014, arXiv:1409.4127.

112. Song, Y.-N.; Zhang, H.-L.; Chang, C.-J.; Bollag, D.M. Cytotoxic Cyclolignans from Koelreuteria henryi. J. Nat. Prod. 1994, 57, 1670-1674. [CrossRef] [PubMed]

113. Chien, S.-C.; Xiao, J.-H.; Tseng, Y.-H.; Kuo, Y.-H.; Wang, S.-Y. Composition and antifungal activity of balsam from Liquidambar formosana Hance. Holzforschung 2013, 67, 345-351. [CrossRef]

114. He, X.; Chen, Y.; Ghamisi, P. Heterogeneous Transfer Learning for Hyperspectral Image Classification Based on Convolutional Neural Network. IEEE Trans. Geosci. Remote. Sens. 2020, 58, 3246-3263. [CrossRef]

115. Vasu, B. Re: Deep Learning - Transfer Learning from One Image Classification Dataset to another-Scientific Process? Available online: https:/ / www.researchgate.net/post/Deep-Learning-transfer-learning-from-one-image-classification-dataset-to-anotherScientific-process/5dcb3691b93ecd25404fb185/citation/download. (accessed on 1 December 2020).

116. Li, X.; Zhang, L.; Du, B.; Zhang, L.; Shi, Q. Iterative Reweighting Heterogeneous Transfer Learning Framework for Supervised Remote Sensing Image Classification. IEEE J. Sel. Top. Appl. Earth Obs. Remote. Sens. 2017, 10, 2022-2035. [CrossRef] 